\title{
FACTORES DE AMORTIGUAMIENTO PARA ESPECTROS DE DESPLAZAMIENTO DE SISTEMAS CON DISIPADORES DE ENERGÍA HISTERÉTICOS
}

\author{
Sonia E. Ruiz Gómez (1) y Miguel A. Orellana Ojeda ${ }^{(2)}$
}

\begin{abstract}
RESUMEN
Se propone y aplica una metodología basada en un análisis probabilista para estimar factores de amortiguamiento $\left(F_{m d}\right)$ que modifican al espectro de desplazamiento elástico. Los $F_{m d}$ toman en cuenta la disipación de energía de los sistemas disipadores del tipo histerético, así como el peligro sísmico del sitio donde se desplantan las estructuras. Se realizan análisis no lineales y lineales en el tiempo para sistemas de un grado de libertad equipados con disipadores de energía (S1GDL duales) y S1GDL convencionales, respectivamente. Se hace ver la influencia en los factores $F_{m d}$ de los distintos parámetros de diseño y las características del suelo. Se obtienen expresiones matemáticas para los $F_{m d}$. los cuales resultan función del periodo de vibración de la estructura, de las características del sistema estructura-disipador y del tipo de suelo (duro, intermedio o blando) en donde se localizan los sistemas estructurales. Las expresiones son útiles para el diseño sísmico preliminar basado en el control de desplazamiento de sistemas estructurales equipados con disipadores del tipo histerético, ubicados en la ciudad de México.
\end{abstract}

Palabras clave: análisis probabilista; disipador de energía histerético; factores de amortiguamiento; espectro de desplazamiento; diseño por desplazamiento

\section{DAMPING FACTORS FOR DISPLACEMENT SPECTRA OF SYSTEMS WITH HYSTERETIC ENERGY DISSIPATERS}

\begin{abstract}
A methodology based on a probabilistic analysis is proposed and applied to estimate damping factors $\left(F_{m d}\right)$ that modify the elastic displacement spectrum. The $F_{m d}$ take into account the energy dissipation of the hysteretic devices, as well as the seismic hazard of the site where the structures are located. Non-Linear and linear time-history analyses are carried out for single-degree-of-freedom systems equipped with energy-dissipation devices (dual S1GDL) and conventional S1GDL, respectively. The influence of the different design parameters and soil characteristics on the $F_{m d}$ factors, is shown. Mathematical expressions are obtained for $F_{m d}$; these depend on the vibration period of the structure, the characteristics of the structure-dissipater system and the type of soil (firm, intermediate or soft)
\end{abstract}

Artículo recibido el 4 de junio de 2019 y aprobado para su publicación el 12 de abril de 2021 . Se aceptarán comentarios y/o discusiones hasta cinco meses después de su publicación.

(1) Investigadora Titular, Instituto de Ingeniería, Universidad Nacional Autónoma de México, Circuito Interior s/n, Ciudad Universitaria, Delegación Coyoacán, México, C.P. 0.4510, Tel. (55)5623-3654. SRuizG@iingen.unam.mx

(2) Estudiante de Posgrado, Instituto de Ingeniería, Universidad Nacional Autónoma de México, Circuito Interior s/n, Ciudad Universitaria, Delegación Coyoacán, México, C.P. 0.4510, Tel. (55)5623-3600. MOrellanaO@iingen.unam.mx

DOI: $10.18867 /$ ris. 105.542 
in which the structural systems are located. The proposed expressions are useful for preliminary seismic design, based on the displacement control of structural systems equipped with hysteretic dissipaters, located in Mexico City.

Keywords: probabilistic analysis; hysteretic energy dissipater; damping factors; displacement spectra; displacement-based design

\section{INTRODUCCIÓN}

La gran mayoría de las estructuras tienen capacidad de disipar energía sísmica a través del amortiguamiento inherente y del comportamiento inelástico de los componentes estructurales. En un sistema convencional, la mayor cantidad de disipación de energía se lleva a cabo a través del comportamiento inelástico del sistema estructural; sin embargo, la incursión en el intervalo inelástico provoca deformaciones excesivas, lo que puede generar daños importantes en los componentes estructurales y no estructurales. Los reglamentos y normas de diseño sísmico comúnmente aceptan que las edificaciones resulten dañadas y/o con deformaciones residuales permanentes después de un sismo intenso, pero sin llegar al colapso, con el fin de proteger la vida de los ocupantes. Por esta razón, se ha impulsado la inclusión de sistemas de protección sísmica en las estructuras a través del uso de disipadores energía, tales como los disipadores histeréticos (Bozzo et al., 2020), cuyo objetivo es reducir la respuesta sísmica (Foti et al., 1998) para disminuir los daños estructurales y no estructurales.

Los disipadores histeréticos están compuestos de un material con capacidad de deformación plástica (p. ej., plomo, acero, cobre, etc.), donde la disipación se activa por medio del desplazamiento relativo entre sus extremos. Entre los principales tipos de disipadores histeréticos se encuentran los tipos TADAS, ADAS, LSB, SLB, Honeycomb, etc; los cuales aumentan la rigidez y la resistencia, modificando las propiedades dinámicas de la estructura. Se debe verificar que los disipadores presenten un comportamiento no lineal ante eventos símicos moderados e intensos para garantizar la disipación de energía; mientras que la estructura principal preferentemente debe permanecer sin daño o con daño mínimo. Por otro lado, ante condiciones de servicio, el comportamiento de los disipadores se debe limitar a presentar comportamiento lineal.

Los disipadores histeréticos son altamente sensibles al desplazamiento, más que a las fuerzas, lo que los hace particularmente apropiados para realizar diseños usando procedimientos basados en el control de desplazamiento (Teran-Gilmore y Virto-Cambray, 2009; Nuzzo et al., 2019; Segovia y Ruiz, 2017; Orellana et al., 2021). Además, el daño estructural ocasionado por eventos sísmicos se relaciona más directamente con los desplazamientos relativos y con las deformaciones de los elementos estructurales, que con las fuerzas (Priestley et al., 2007).

La mayoría de los reglamentos de construcción, incluyendo el Reglamento de Construcciones del Distrito Federal (RCDF-20, 2020), permite una reducción de las ordenadas espectrales de diseño por medio de factores de ductilidad, de sobrerresistencia y de amortiguamiento. Este último puede ser proporcionado por el efecto interacción suelo-estructura (Avilés y Pérez-Rocha, 1996) o por amortiguamiento suplementario (Castillo y Ruiz, 2013, 2014). El diseño de edificios, en la mayor parte del mundo, se basa en el método de las fuerzas, revisando que los desplazamientos no excedan cierto límite tolerable, para diferentes estados límite (p. ej., servicio y seguridad de vida).

Existen diferentes estudios que proponen expresiones para reducir las ordenadas espectrales sísmicas debido al amortiguamiento suplementario proporcionado a la estructura, por ejemplo: Del Valle, 1988; Naeim y Kircher, 2001; Arroyo-Espinoza y Terán-Gilmore, 2002; Bommer y Mendis, 2004; Cameron y Green, 2007; Hidalgo y Ruiz, 2010; Castillo y Ruiz, 2014. Estas expresiones son aplicables principalmente 
a sistemas con disipadores viscosos; sin embargo, existen muy pocas expresiones en la literatura que sean aplicables a sistemas con disipadores histeréticos (cuya disipación de energía depende del desplazamiento entre sus extremos), y que, además, sean útiles para el diseño sísmico basado en desplazamiento.

En la literatura existen trabajos que proponen factores de modificación de sobrerresistencia y ductilidad para sistemas equipados con disipadores histeréticos (Asgarian y Shokrgozar, 2009; Abdollahzadeh et al., 2012). Jara et al. (2000) estudian la respuesta de sistemas de un grado de libertad equipados con disipadores de energía del tipo histerético con comportamiento elastoplástico; sin embargo, estos estudios se aplican a un limitado número de sistemas, y no proponen factores de modificación al espectro de desplazamiento.

Tomando en cuenta las limitaciones que hay en los trabajos antes mencionados, en el presente estudio se obtienen expresiones matemáticas para estimar factores de amortiguamiento para modificar los espectros de desplazamiento de diseño sísmico. Se considera el efecto del disipador de tipo histerético, así como las características dinámicas del suelo donde se localizan las estructuras. Esto último, debido a que la eficiencia de los disipadores varía según las características (duración efectiva y periodo dominante) del movimiento del suelo. Las expresiones matemáticas se obtienen a partir de cocientes entre Espectros de Tasa de Excedencia Uniforme (ETEU) de desplazamiento correspondientes a sistemas estructurales de un grado de libertad (S1GDL) con disipadores histeréticos (sistemas duales), y ETEU correspondientes a sistemas sin disipadores (sistemas convencionales), ubicados en distintas zonas de la ciudad de México (CDMX).

\section{ECUACIONES QUE GOBIERNAN A LOS S1GDL EQUIPADOS CON DISIPADORES DE TIPO HISTERÉTICO}

\section{Sistema simple de un grado de libertad}

La respuesta sísmica de un S1GDL, considerando comportamiento no-lineal, se puede estimar partir de la siguiente ecuación de movimiento (Sues et al., 1988):

$m \ddot{u}+c \dot{u}+\delta k u+(1-\delta) k z=-m \ddot{u}_{g}(t)$

donde $m$ es la masa, $c$ es el coeficiente de amortiguamiento viscoso y $k$ es la rigidez lateral del sistema. Los términos $\ddot{u}, \dot{u}$ y $u$ son la aceleración, velocidad y desplazamiento de la masa con respecto a la base, respectivamente. El término $\ddot{u}_{g}(t)$ es la aceleración en la base del sistema y $z$ es la componente histerética con unidades de desplazamiento. Los términos $\delta k u$ y $(1-\delta) k z$ representan la fuerza restauradora que depende del desplazamiento $u$ y de la componente histerética $z$, respectivamente. La componente histerética se obtiene al modelar los ciclos histeréticos por medio de una ecuación diferencial de primer orden. Esta ecuación diferencial, capaz de considerar degradación del material, está dada por (Bouc, 1967; Baber y Wen, 1981):

$\dot{z}=\frac{A \dot{u}-v\left(\beta|\dot{u}||z|^{n-1} z+\lambda \dot{u}|z|^{n}\right)}{\eta}$

donde $A, \beta, \lambda$ y $n$ son parámetros que controlan la amplitud, forma del ciclo histerético, y la suavidad de la transición entre el intervalo elástico y el inelástico; $\eta$ y $v$ son parámetros que controlan el deterioro de la rigidez y de la resistencia, respectivamente. El parámetro $\delta$ en la ec. 1 representa la relación entre la rigidez post-fluencia y la rigidez inicial del sistema, $k$, cuando $A=1$. 
Para modelos no lineales de elementos de acero se puede considerar que $\beta=\lambda$, y para elementos de concreto $\beta=-3 \lambda$ (Sues et al., 1988). Para el caso de elementos de acero, el parámetro $\lambda$ se obtiene a partir de la siguiente ecuación (Silva-González, 1998):

$\lambda=\frac{1}{2 v}\left(\frac{k}{V_{y}}\right)^{n}$

donde $V_{y}$ es la fuerza a partir de la cual fluye un sistema elastoplástico perfecto.

Para obtener la historia de desplazamientos, velocidades y aceleraciones del sistema en el tiempo ante una excitación sísmica, se resuelve el sistema de ecuaciones diferenciales formado por la ec. 1 y la ec. 2 utilizando algún método numérico (p. ej., Runge-Kutta de cuarto orden).

\section{Sistema dual de un grado de libertad}

El sistema dual o estructura-disipador (figura 1a) se considera formado por un sistema estructural primario (por lo regular son marcos resistentes a momento flexionante que son capaces de resistir al menos las fuerzas gravitacionales), más un sistema secundario compuesto por elementos disipadores de energía que, en general, no se diseña para resistir fuerzas gravitacionales, sino fuerzas laterales, y deben disipar importantes cantidades de energía a través de su fluencia en ambos sentidos (en el caso de disipadores del tipo histerético). Estos dispositivos pueden verse sometidos a fuerzas debidas a cargas gravitacionales, (dependiendo del tipo de disipador histerético); sin embargo, en el presente estudio se supone que las fuerzas laterales provocadas por eventos sísmicos son las que rigen su diseño. Además, se considera que un comportamiento estructural deseable es que el sistema primario no incursione en el intervalo inelástico, y que la disipación de energía se concentre en el sistema secundario.

El modelo correspondiente para un sistema dual se puede representar de manera simplificada como un S1GDL más un elemento secundario en paralelo que representa al disipador de energía sísmica (Nakashima et al., 1996; Ruiz y Badillo, 2001; Guerrero et al., 2016), como se muestra en la figura 1b. En la figura 2 se muestran esquemáticamente curvas de capacidad de los sistemas primario, secundario y dual.

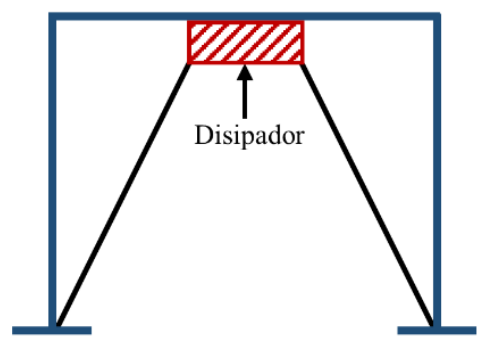

a) Estructura dual

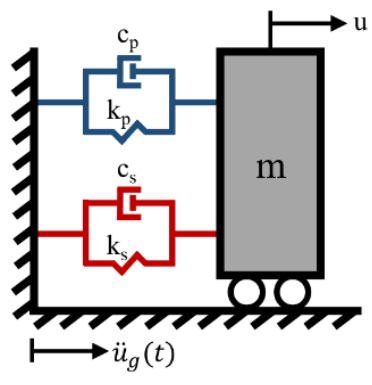

b) S1GDL dual

Figura 1. Modelo de un sistema dual 


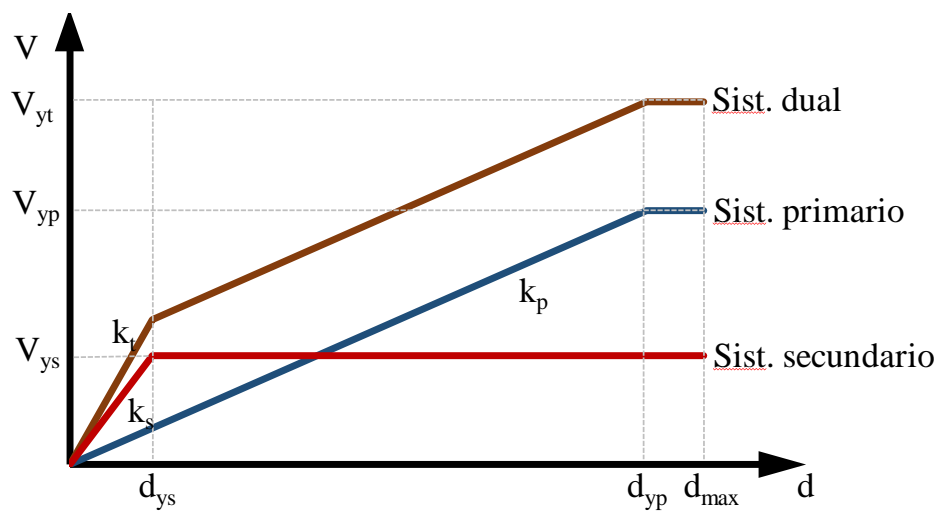

Figura 2. Curvas de capacidad

En la figura 2 el término $k$ representa la rigidez lateral, y $V$ es la resistencia del sistema. Los subíndices $p, s$ y $t$ se refieren al sistema primario, al sistema secundario y al sistema dual, respectivamente; el subíndice $y$ indica la fluencia de los sistemas, y $d_{\max }$ es el desplazamiento máximo.

La relación de rigideces y de resistencias del sistema dual se obtiene mediante los parámetros $\alpha$ y $\gamma$, respectivamente. La relación de rigideces $\alpha$ es la relación entre la rigidez del sistema primario $k_{p}$ y la rigidez del sistema dual $k_{t}$ :

$\alpha=\frac{k_{p}}{k_{t}}$

La relación de rigideces también se puede representar como $\alpha=\left(T_{t} / T_{p}\right)^{2}$, donde $T_{p}$ es el periodo del sistema primario y $T_{t}$ es el periodo del sistema dual. Por otra parte, la relación de resistencias $\gamma$ se define como la relación entre la fuerza de fluencia del disipador $V_{y s}$ y la fuerza de fluencia del sistema dual $V_{y t}$ :

$\gamma=\frac{V_{y s}}{V_{y t}}$

Las propiedades de rigidez y de resistencia del sistema dual se obtienen al sumar las propiedades del sistema principal con las del sistema disipador de energía (sistema secundario). De esta forma, el valor de la rigidez total y el de la fuerza total del sistema son, respectivamente:

$k_{t}=k_{p}+k_{s}$

$V_{y t}=V_{y p}+V_{y s}$

Al sustituir la ec. 7 en ec. 5 se puede encontrar $V_{y s}$ en función de $\gamma$ y de $V_{y p}$, de la siguiente manera:

$V_{y s}=\frac{V_{y p} \gamma}{1-\gamma}$

Por otra parte, a partir de la ec. 1 y de la figura $1 \mathrm{~b}$, la respuesta de un sistema de un grado de libertad dual (S1GDL dual) ante una excitación sísmica, considerando comportamiento no lineal en el sistema convencional y en el disipador de energía, se puede estimar a partir de la siguiente ecuación de movimiento: 
$m \ddot{u}+\left(c_{p}+c_{s}\right) \dot{u}+\delta_{p} k_{p} u+\left(1-\delta_{p}\right) k_{p} z_{p}+\delta_{s} k_{s} u+\left(1-\delta_{s}\right) k_{s} z_{s}=-m \ddot{u}_{g}(t)$

Para este caso, la fuerza restauradora está dada por la contribución de la fuerza restauradora del sistema principal (términos con subíndice $p$ ) más la del disipador (términos con subíndice $s$ ).

Las componentes histeréticas se obtienen al modelar los ciclos histeréticos por medio de una ecuación diferencial de primer orden. Las ecuaciones diferenciales del sistema convencional y del disipador son, respectivamente (Bouc, 1967; Baber y Wen, 1981):

$\dot{z}_{p}=\frac{\mathrm{A}_{p} \dot{u}-v_{p}\left(\beta_{p}|\dot{u}|\left|z_{p}\right|^{\mathrm{n}_{p}-1} z_{p}+\lambda_{p} \dot{u}\left|z_{p}\right|^{\mathrm{n}_{p}}\right)}{\eta_{p}}$
$\dot{z}_{S}=\frac{\mathrm{A}_{s} \dot{u}-v_{s}\left(\beta_{s}|\dot{u}|\left|z_{s}\right|^{\mathrm{n}_{s}-1} z_{s}+\lambda_{s} \dot{u}\left|z_{s}\right|^{\mathrm{n}_{s}}\right)}{\eta_{s}}$

Los términos $A, \beta, \lambda, n, \eta \mathrm{y} v$ tienen el mismo significado que en la ec. 2. El comportamiento histerético de los disipadores se considera distinto al del sistema principal; por ello, existen dos ecuaciones constitutivas: una para modelar el comportamiento del sistema principal y otra para el sistema disipador.

Para obtener la historia de desplazamientos, velocidades y aceleraciones del sistema en el tiempo ante una excitación sísmica, se resuelve el sistema de ecuaciones diferenciales formado por las ecs. 9 a 11 utilizando algún método numérico.

\section{Parámetros de diseño}

En los análisis del S1GDL dual se considera que el sistema principal tiene comportamiento lineal; por lo tanto, los parámetros que se utilizan son $A_{p}=n_{p}=\eta_{p}=v_{p}=\delta_{p}=1$. Para el sistema secundario se considera comportamiento no lineal (sin degradación de rigidez ni resistencia) con parámetros $A_{s}=\eta_{s}=v_{s}=1$; transición del intervalo elástico al inelástico de forma suave (característica importante en los disipadores del tipo histerético) dado por $n_{s}=1$; y relación de rigidez post-fluencia con la rigidez elástica de $\delta_{s}=0.025$. Los parámetros $n_{s}$ y $\delta_{s}$ se eligieron con base en estudios experimentales de Hurtado y Bozzo (2008), y Cameron et al. (2004). Además, se considera que $\beta_{s}=\lambda_{s}$, con base en recomendaciones de Sues et al. (1988).

En las figuras $3 \mathrm{a}$ y $3 \mathrm{~b}$ se muestra el comportamiento típico de los ciclos histeréticos para el sistema principal y secundario, respectivamente, donde $f_{s}$ es la fuerza restauradora.

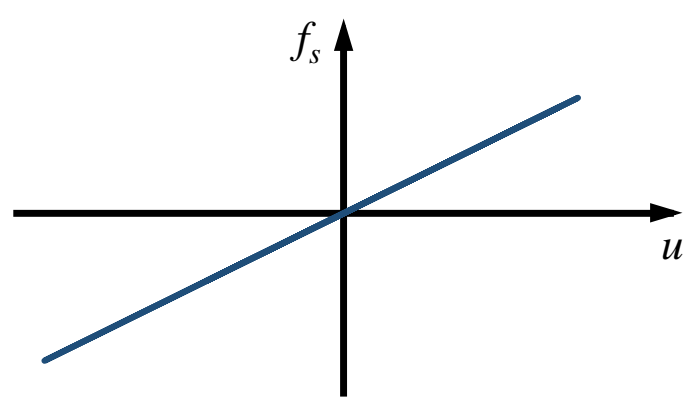

a) Sistema principal

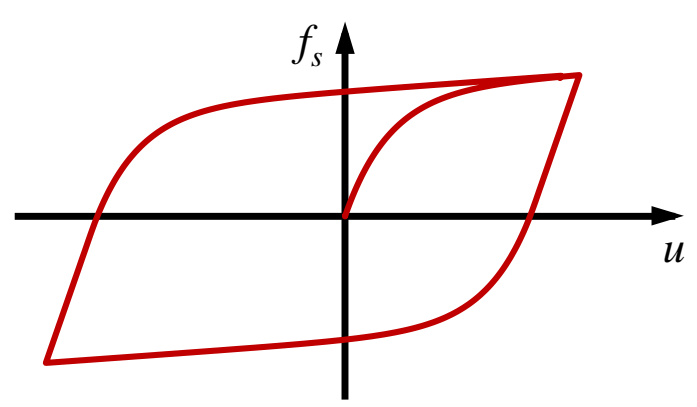

b) Sistema secundario

Figura 3. Comportamiento de los ciclos histeréticos del sistema principal y del secundario del S1GDL dual 
En el presente estudio se analizan valores de relación de rigideces $0.25 \leq \alpha \leq 0.60$; el límite inferior del intervalo se elige para evitar tener dimensiones excesivamente grandes en los disipadores, y el límite superior para que haya una contribución significante de rigidez del sistema disipador al sistema total (Vargas y Bruneau, 2009; Nangullasmú, 2011; Segovia y Ruiz, 2017). El límite inferior de la relación de resistencias $\gamma$ se elige con base en recomendaciones de Segovia y Ruiz (2017).

La ductilidad del sistema disipador está dada por:

$\mu_{s}=\frac{d_{\max }}{d_{y s}}$

A partir de las ecs. 4 a 7 y la ec. 12, considerando comportamiento lineal del sistema principal $\left(d_{\max }=d_{y p}\right)$, se puede expresar la ductilidad que desarrolla el sistema disipador en función de los parámetros $\alpha$ y $\gamma$ de la siguiente forma:

$\mu_{s}=\frac{(1-\alpha)(1-\gamma)}{\alpha \gamma}$

En la ec. 13 se puede ver que entre más grande sea el valor de $\alpha$ y $\gamma$, se tienen menores demandas de ductilidad en el disipador.

En el presente estudio, para asegurar que el sistema secundario disipe energía, se considera que el disipador desarrolla ductilidades $\mu_{s} \geq 1.5$. Tomando en cuenta lo anterior, en la tabla 1 se muestran los valores de $\alpha$ y $\gamma$ que se utilizan en los análisis del presente estudio. En la tabla 1 se puede notar que el intervalo de valores $\gamma$ es diferente para cada valor de $\alpha$.

Tabla 1. Valores de $\alpha$ y $\gamma$ utilizados en este estudio

\begin{tabular}{cc}
$\alpha$ & $\gamma$ \\
\hline 0.25 & $0.25-0.65$ \\
0.30 & $0.20-0.60$ \\
0.35 & $0.20-0.55$ \\
0.40 & $0.20-0.50$ \\
0.45 & $0.20-0.45$ \\
0.50 & $0.20-0.40$ \\
0.55 & $0.20-0.35$ \\
0.60 & $0.20-0.30$ \\
\hline
\end{tabular}

\section{METODOLOGÍA GENERAL}

En el presente trabajo se propone una metodología basada en un análisis probabilista que considera el peligro sísmico del sitio, con el fin de estimar factores de modificación del espectro desplazamiento. Para ello, se emplean los siguientes pasos:

1. Se obtiene la respuesta sísmica de un gran número de S1GDL correspondiente a sistemas duales equipados con disipadores de energía del tipo histerético (S1GDL dual) y, alternativamente, a sistemas convencionales sin disipadores (S1GDL simple). Se consideran diferentes combinaciones en las características del sistema dual y diferentes periodos de vibración. Las características del sistema dual están en función de la relación de rigideces $\alpha$ y de resistencias $\gamma$. Se realizan análisis (no 
lineales y lineales) en el tiempo para calcular el Parámetro de Demanda Estructural (PDE) en función de la Medida de Intensidad (MI) sísmica tanto para el S1GDL dual como para el S1GDL simple. Para el S1GDL dual se considera que la no linealidad se presenta únicamente en el sistema secundario (disipador).

2. Se obtiene la mediana $\left(\mu_{\mathrm{lnPDE}}\right)$ y la desviación estándar $\left(\sigma_{\mathrm{lnPDE}}\right)$ del logaritmo natural del PDE, para cada MI.

3. Con las estadísticas del PDE, se calculan las curvas de fragilidad, correspondientes a ciertos valores específicos del PDE, usando la siguiente ecuación (Esteva, 1967):

$$
P(P D E>y \mid M I=m i)=1-\Phi\left(\frac{\ln \left(\frac{y}{\mu_{\ln P D E}}\right)}{\sigma_{\ln P D E}}\right)
$$

4. Para los diferentes sistemas equipados con disipadores de energía, así como para los sistemas convencionales, se estiman las tasas de excedencia media anual para ciertos valores específicos del PDE (curva de excedencia de demanda estructural) con la siguiente ecuación (Esteva, 1967; Cornell, 1968):

$v_{P D E}(y)=\int\left|\frac{d v_{M I}(m i)}{d(m i)}\right| P(P D E>y \mid M I=m i) d m i$

donde $v_{M I}(m i)$ es el número medio de veces por año que ocurre una intensidad mayor o igual que $m i$, con este valor se genera la curva de peligro sísmico del sitio de interés asociado al periodo de vibrar del sistema estructural $\left(\mathrm{T}_{1}\right) ; P(P D E>y \mid M I=m i)$ es la curva de fragilidad, la cual representa la probabilidad condicional de que el valor del $P D E$ exceda el valor, $y$, dada una intensidad $M I=m i$. En este estudio se considera como medida de intensidad sísmica (MI) a la seudoaceleración (Sa), y como parámetro de demanda estructural (PDE) al valor del desplazamiento espectral (D), asociados al periodo de vibrar del sistema estructural $\left(\mathrm{T}_{1}\right)$.

5. A partir de las curvas de peligro de demanda estructural se construyen Espectros de Tasa de Excedencia Uniforme (ETEU) de desplazamientos para una variedad de sistemas convencionales y sistemas duales con diferentes características. Los ETEU corresponden a un periodo de recurrencia de 250 años.

6. Con la finalidad de obtener los factores de modificación de las ordenadas espectrales de desplazamiento, se obtienen relaciones entre ETEU de desplazamientos correspondientes a sistemas duales y ETEU de sistemas convencionales.

7. Finalmente, se ajustan expresiones matemáticas correspondientes a los cocientes espectrales. Las expresiones propuestas están en función del periodo de vibrar del sistema, del periodo de vibrar del suelo, así como de las relaciones de rigideces $\alpha$ y de relaciones de resistencias $\gamma$ del sistema dual.

\section{MOVIMIENTOS SÍSMICOS}

Para este estudio se utilizaron 360 movimientos sísmicos registrados en diferentes estaciones localizadas en la ciudad de México, correspondientes a la red acelerográfica del CIRES (Centro de Instrumentación y Registro Sísmico A. C.) y de la RAII-UNAM (Instituto de Ingeniería de la Universidad Nacional Autónoma de México). Los movimientos sísmicos corresponden a 18 eventos sísmicos de subducción con magnitudes mayores o iguales que 5.9, profundidades focales menores a $40 \mathrm{~km}$ y distancias epicentrales menores a $700 \mathrm{~km}$. En la tabla 2 se muestra las características principales de los eventos sísmicos utilizados (Global CMT), y en la figura 4 se muestra la ubicación de sus epicentros. 
A cada movimiento sísmico, se realizó una corrección de línea base del tipo normal restando una constante (promedio de aceleraciones) a todo el acelerograma para lograr un promedio de aceleraciones igual a cero (Ordaz y Montoya, 2014) y un filtro "bandpass" del tipo Butterworth de orden 4 con frecuencias de corte de 0.1 a $25 \mathrm{~Hz}$ y 0.1 a $10 \mathrm{~Hz}$ para acelerogramas con intervalo de muestreo $\Delta \mathrm{t} \leq 0.02 \mathrm{~s}$ y $\Delta \mathrm{t} \geq 0.03$ $\mathrm{s}$, respectivamente; las frecuencias de corte se seleccionaron para tener un valor promedio de la historia de desplazamiento de suelo aproximadamente igual a cero y para que las ordenadas a periodos largos en el espectro de desplazamiento correspondieran al desplazamiento máximo de suelo. Además, se consideró una duración del movimiento sísmico correspondiente al 2.5\% y $97.5 \%$ de la intensidad de Arias.

Tabla 2. Características de los eventos sísmicos

\begin{tabular}{cccccc}
\hline \multirow{2}{*}{ Evento } & \multirow{2}{*}{ Fecha } & \multirow{2}{*}{ Magnitud } & \multicolumn{2}{c}{ Coordenadas del epicentro } & \multirow{2}{*}{$\begin{array}{c}\text { Profundidad } \\
\text { (km) }\end{array}$} \\
\hline 1 & $19 / 09 / 1985$ & 8.0 & 17.91 & 101.99 & 21.3 \\
2 & $21 / 09 / 1985$ & 7.5 & 17.57 & 101.42 & 20.8 \\
3 & $30 / 04 / 1986$ & 6.9 & 18.25 & 102.92 & 20.7 \\
4 & $25 / 04 / 1989$ & 6.9 & 16.83 & 99.12 & 15.0 \\
5 & $31 / 05 / 1990$ & 5.9 & 16.77 & 100.12 & 26.0 \\
6 & $15 / 05 / 1993$ & 6.0 & 16.45 & 97.92 & 38.5 \\
7 & $24 / 10 / 1993$ & 6.6 & 16.77 & 98.61 & 21.8 \\
8 & $14 / 09 / 1995$ & 7.3 & 16.73 & 98.54 & 21.8 \\
9 & $09 / 10 / 1995$ & 8.0 & 19.34 & 104.80 & 15.0 \\
10 & $15 / 07 / 1996$ & 6.6 & 17.50 & 101.12 & 22.4 \\
11 & $03 / 02 / 1998$ & 6.3 & 15.92 & 96.22 & 24.0 \\
12 & $09 / 08 / 2000$ & 6.5 & 18.13 & 102.39 & 33.0 \\
13 & $22 / 01 / 2003$ & 7.5 & 18.86 & 103.90 & 26.0 \\
14 & $20 / 03 / 2012$ & 7.5 & 16.60 & 98.39 & 15.4 \\
15 & $11 / 04 / 2012$ & 6.7 & 18.10 & 102.97 & 20.5 \\
16 & $18 / 04 / 2014$ & 7.3 & 17.55 & 101.25 & 18.9 \\
17 & $08 / 05 / 2014$ & 6.5 & 17.36 & 100.74 & 21.3 \\
18 & $10 / 05 / 2014$ & 6.1 & 17.31 & 100.82 & 20.7 \\
\hline
\end{tabular}

Para cada movimiento sísmico se calculó el espectro elástico de seudoaceleración considerando 5\% del amortiguamiento crítico y se determinó de manera aproximada su periodo dominante. El periodo dominante en este estudio se define como el periodo en donde se presenta la máxima ordenada del espectro de seudoaceleración, el cual depende del tipo de suelo en donde se registra el movimiento. Los movimientos sísmicos se clasificaron en siete zonas de acuerdo con el periodo dominante y la ubicación de la estación sísmica donde se obtuvo el registro. La tabla 3 muestra las siete zonas consideradas dentro de la ciudad de México con sus respectivos intervalos de periodos dominantes del suelo, que son las mismas zonas que se mencionan en las Normas Técnicas Complementarias para Diseño por Sismo (2017) del Reglamento de Construcciones del Distrito Federal (2017). 


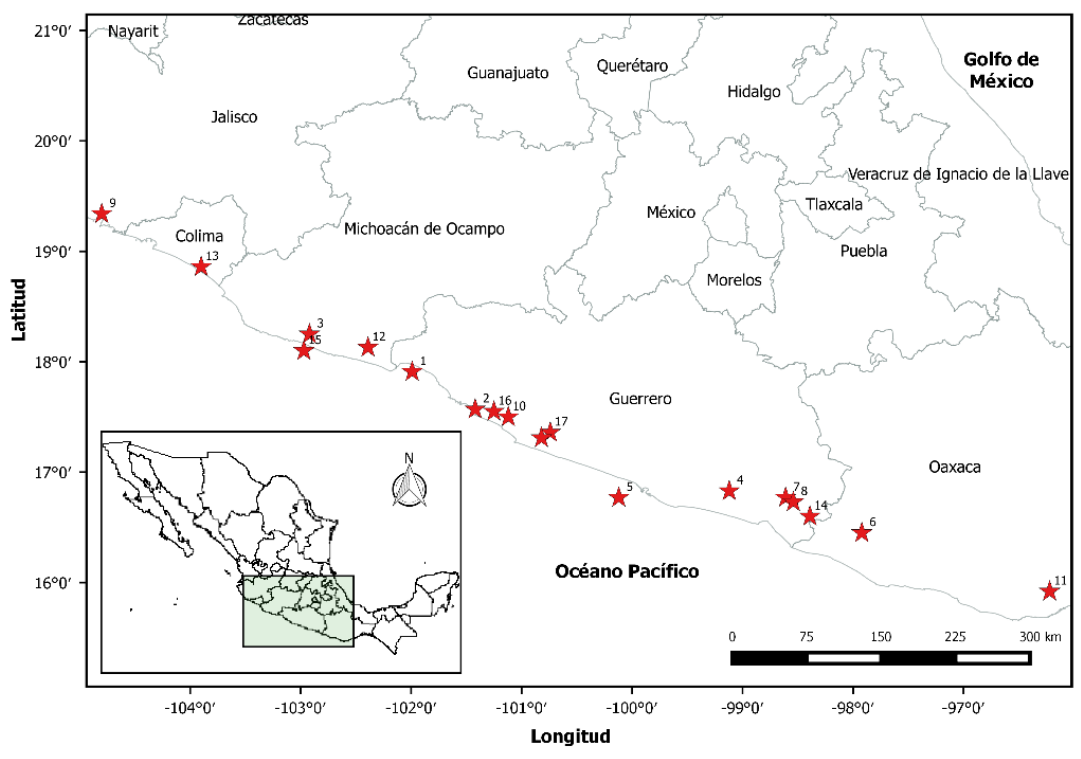

Figura 4. Epicentros de los sismos utilizados en este estudio

Tabla 3. Zonas sísmicas

\begin{tabular}{cc}
\hline Zona & Periodo dominante del suelo $\left(\mathrm{T}_{\mathrm{s}}\right)$ \\
\hline A & $0<\mathrm{T}_{\mathrm{s}} \leq 0.5$ \\
$\mathrm{~B}$ & $0.5<\mathrm{T}_{\mathrm{s}} \leq 1.0$ \\
$\mathrm{C}$ & $1.0<\mathrm{T}_{\mathrm{s}} \leq 1.5$ \\
$\mathrm{D}$ & $1.5<\mathrm{T}_{\mathrm{s}} \leq 2.0$ \\
$\mathrm{E}$ & $2.0<\mathrm{T}_{\mathrm{s}} \leq 2.5$ \\
$\mathrm{~F}$ & $2.5<\mathrm{T}_{\mathrm{s}} \leq 3.0$ \\
$\mathrm{G}$ & $3.0<\mathrm{T}_{\mathrm{s}} \leq 4.0$ \\
\hline
\end{tabular}

Las figuras 5 a a $5 \mathrm{~g}$ muestran los espectros elásticos de desplazamiento, considerando un $5 \%$ del amortiguamiento crítico, correspondientes a cada zona sísmica. En las figuras se observan las grandes diferencias de demanda de desplazamiento que existe entre una zona y otra. Las menores demandas de desplazamiento elástico se presentan en las zonas A, B y C, correspondientes a suelo firme formado por roca, intermedio formado por estratos arenosos limosos, y suelos con estratos de arcilla poco profundos, respectivamente; mientras que las mayores demandas de desplazamiento se presentan en las zonas D, E, F y G, correspondientes a suelos blandos formados por depósitos de arcilla altamente compresibles con espesores superiores a los $50 \mathrm{~m}$. Si se comparan los desplazamientos espectrales máximos de la zona E con los de la zona A, las diferencias entre las demandas de desplazamiento pueden llegar a ser hasta 8 veces mayores en las zonas blandas que en la zona firme.

En la figura 5 también se observa que los espectros de desplazamiento para las zonas C, D, E, F y G, tienen un pico muy bien marcado, es decir, las demandas de desplazamientos se incrementan hasta alcanzar la mayor ordenada espectral y después tienen a disminuir hasta alcanzar el desplazamiento máximo del suelo; para estas zonas, el periodo donde se alcanza la mayor demanda de desplazamiento se encuentra dentro del intervalo de periodos dominantes del sitio (ver tabla 3). Los espectros de las zonas A y B tienen un comportamiento distinto, debido a que tienen uno o más picos y el periodo donde se alcanza la mayor demanda de desplazamiento no se encuentra dentro del intervalo de periodos dominantes del sitio. Lo anterior hace ver la influencia que tiene el tipo de suelo en las demandas de desplazamiento elástico de las estructuras. 


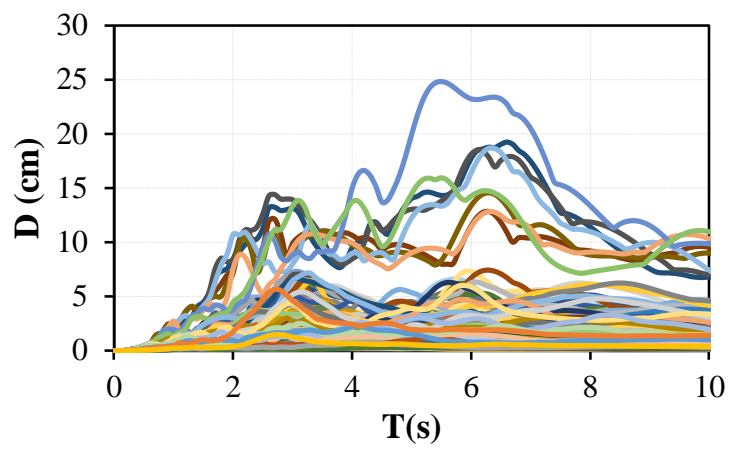

a) Zona $\mathrm{A}$

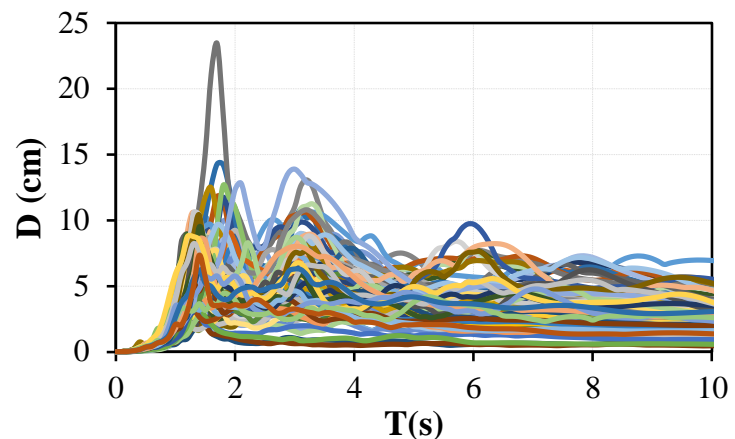

c) Zona C

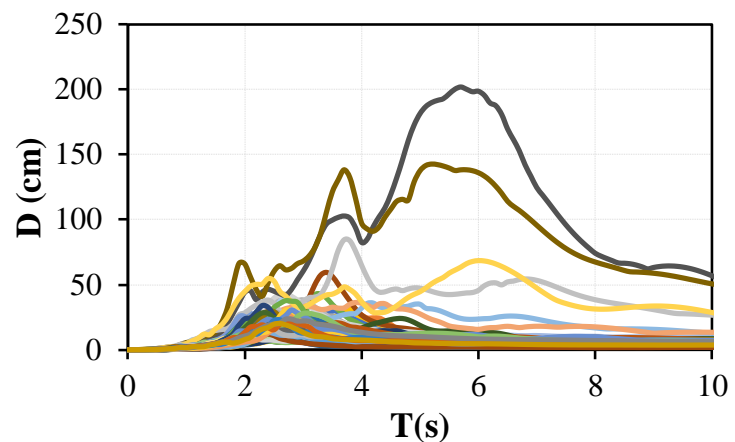

e) Zona E

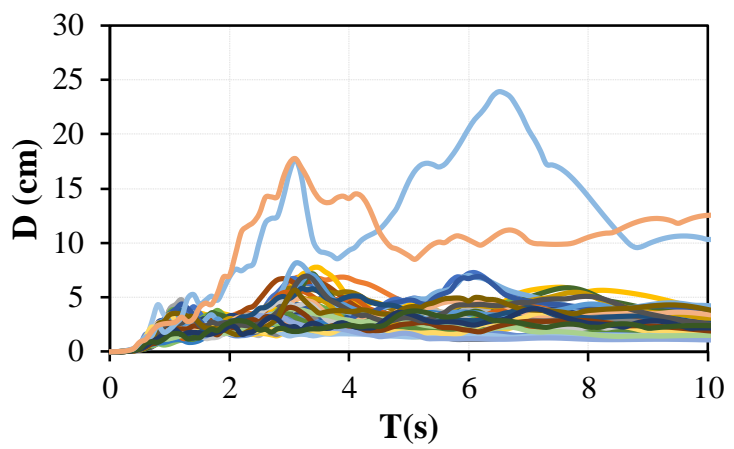

b) Zona B

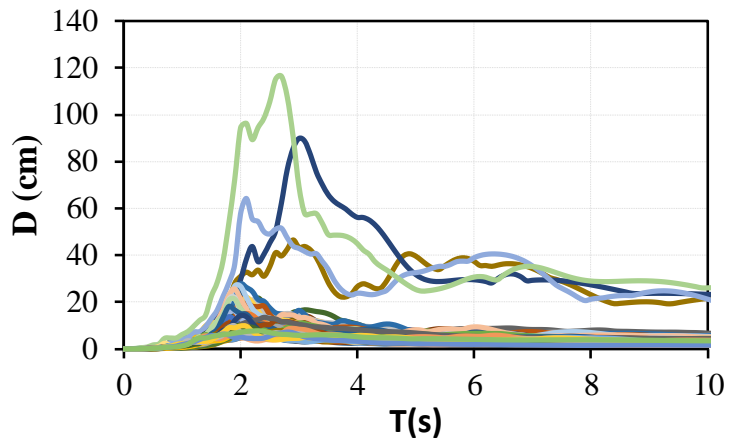

d) Zona D

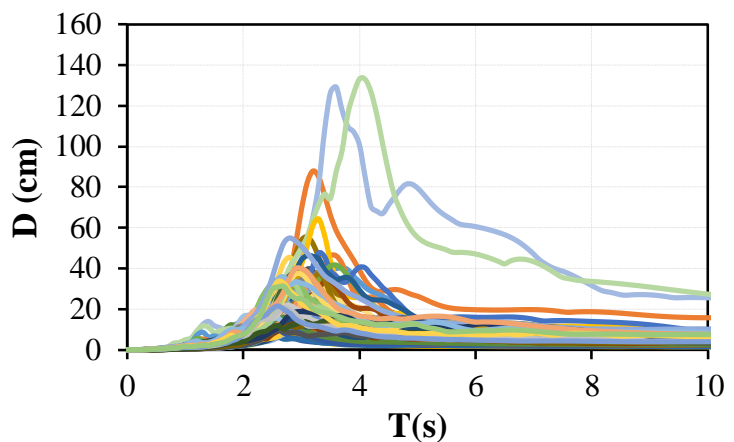

f) Zona $F$

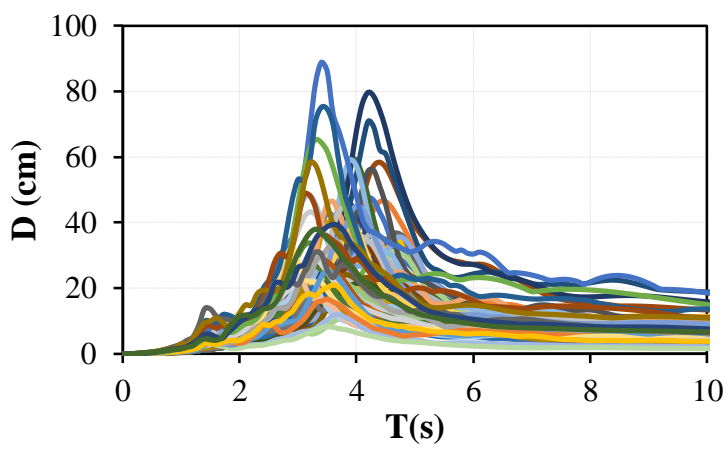

g) Zona G

Figura 5. Espectros elásticos de desplazamiento $(\xi=5 \%)$ 


\section{CURVAS DE PELIGRO SÍSMICO}

Las curvas de peligro sísmico se obtienen de un análisis probabilista y se expresan por lo general en términos de tasas de excedencia anual de un cierto tipo de intensidad. Estas curvas indican el número medio de veces por año que ocurren valores de intensidad sísmica mayores o iguales que un valor de intensidad dado.

Para este estudio se utilizaron las curvas de peligro sísmico obtenidas por Castillo y Ruiz (2013), las cuales consideran como intensidad sísmica al valor de seudoaceleración espectral ( $\mathrm{Sa}$ ) asociado al periodo de vibrar del sistema estructural $\left(\mathrm{T}_{1}\right)$. Las curvas de peligro sísmico se obtuvieron de utilizar 334 movimientos sísmicos de subducción registrados en distintas estaciones localizadas en la ciudad de México correspondientes a sismos con magnitud mayor a 6.9. Como ejemplo, en la figura 6 se muestra una curva de peligro sísmico asociada a un periodo estructural $\mathrm{T}_{1}=2 \mathrm{~s}$, el cual se encuentra dentro del intervalo de periodos dominantes del suelo de la zona $\mathrm{D}$ (ver tabla 3 ).

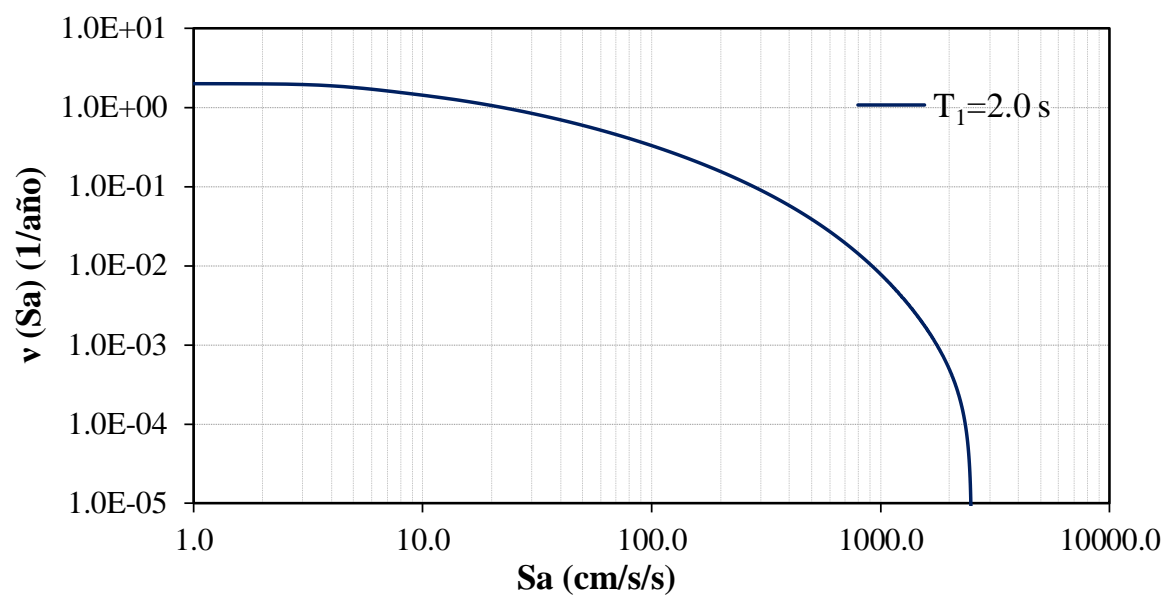

Figura 6. Curva de peligro sísmico correspondiente a la zona D

\section{INFLUENCIA DE LOS PARÁMETROS DE DISEÑO Y DEL TIPO DE SUELO}

En las secciones que siguen se hace ver la influencia de los parámetros de diseño, así como la influencia del tipo de suelo en: 1) las curvas de fragilidad, 2) las curvas de excedencia de desplazamiento, 3) espectros de tasa de excedencia uniforme (ETEU), y 4) cocientes de ETEU de desplazamiento.

\section{Influencia del tipo de suelo en las curvas de fragilidad}

Para obtener las curvas de excedencia de demanda estructural es necesario calcular las curvas de fragilidad (ver ec. 14). En este estudio se considera una distribución de probabilidad del tipo lognormal para la respuesta estructural (Rosenblueth y Esteva, 1972; Shome y Cornell, 1999).

En las figuras 7 a y $7 \mathrm{~b}$ se muestran algunas curvas de fragilidad para demandas de desplazamiento de 50 y $100 \mathrm{~cm}$ para sistemas duales con $T_{1}=2 \mathrm{~s}, \alpha=0.30$ y $\gamma=0.40$ correspondientes a la zona $A$ y a la zona $D$, respectivamente. En las figuras se puede observar que la probabilidad de exceder un cierto valor dado de demanda de desplazamiento, para cierta intensidad, es mucho mayor para los sistemas ubicados en la zona A que para los sistemas ubicados en la zona D; es decir, en la zona A (suelo firme) los sistemas tienen demandas de desplazamiento mucho menores que en la zona $\mathrm{D}$ (suelo blando). 
En las figuras $8 \mathrm{a}$ y $8 \mathrm{~b}$ se muestran curvas de fragilidad para una demanda de desplazamiento de 50 cm para sistemas duales con $T_{1}=2 \mathrm{~s}, \alpha=0.30$ y diferentes valores de $\gamma$ correspondientes a la zona A y a la zona $\mathrm{D}$, respectivamente. En las figuras se puede observar que para los sistemas ubicados en la zona A (suelo firme) la probabilidad de exceder cierto valor de demanda de desplazamiento disminuye conforme el valor de $\gamma$ aumenta en general para los diferentes niveles de intensidad sísmica, mientras que en la zona D (suelo blando) sucede lo contrario. De esta forma se hace notar la influencia que tienen las características dinámicas (periodo dominante) del suelo en donde se ubica la estructura, así como la duración del movimiento sísmico, en la eficiencia de los disipadores para controlar los desplazamientos.

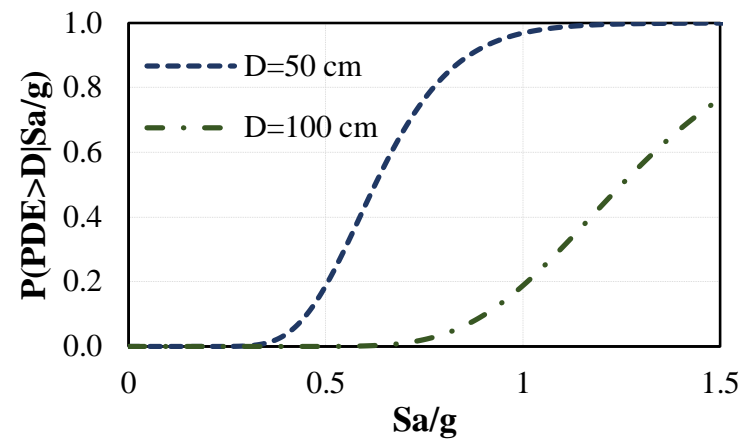

a) Zona $\mathrm{A}$

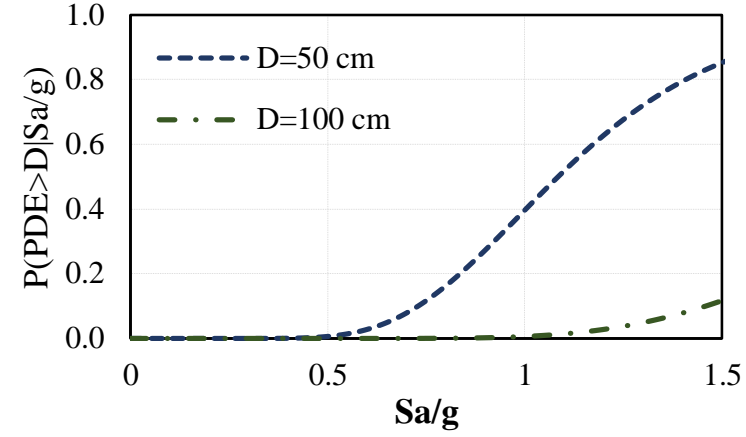

b) Zona D

Figura 7. Curvas de fragilidad para S1GDL dual con $\mathrm{T}_{1}=2 \mathrm{~s}, \alpha=0.30$ y $\gamma=0.40$

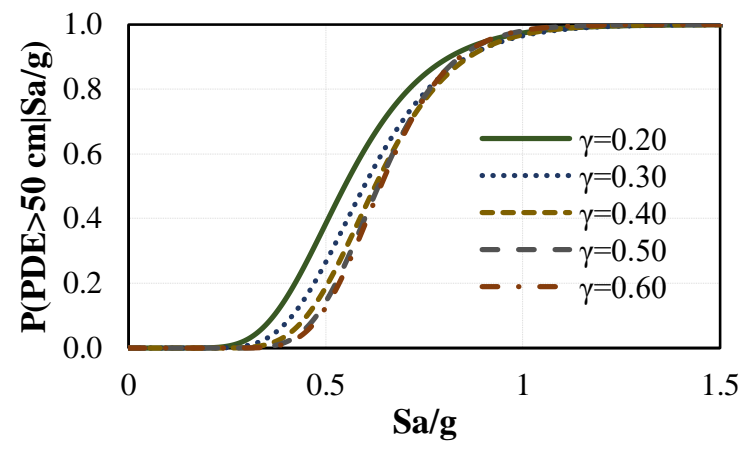

a) Zona $\mathrm{A}$

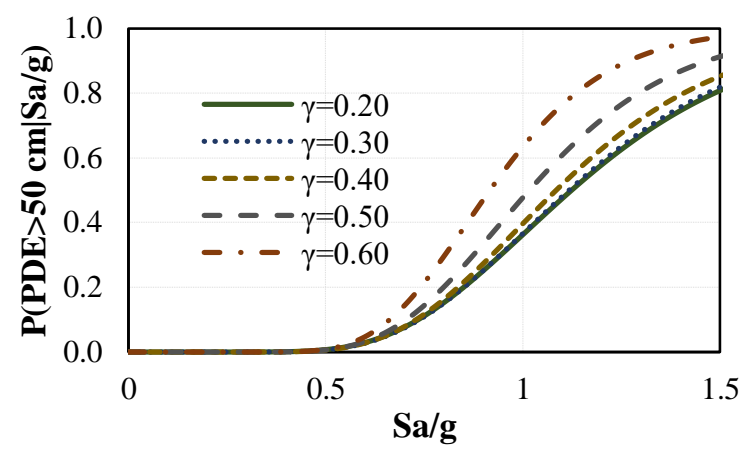

b) Zona D

Figura 8. Curvas de fragilidad para S1GDL dual con $T_{1}=2 \mathrm{~s}, \alpha=0.30$, y diferentes valores de $\gamma$

\section{Influencia del tipo de suelo en las curvas de excedencia de desplazamiento}

Las curvas de excedencia media anual se expresan en términos de tasas de excedencia de cierto parámetro de demanda estructural y definen el número medio de veces por año que se excede un cierto valor de respuesta estructural. En este estudio se elige como respuesta estructural al valor del desplazamiento espectral asociado al periodo de vibrar del sistema estructural (D, $\left.\mathrm{T}_{1}\right)$.

Una vez que se conocen las curvas de fragilidad y las curvas de peligro sísmico, se aplica la ec. 15 para estimar la tasa anual de excedencia de la respuesta estructural, lo cual permite generar la curva de excedencia media anual de desplazamiento correspondiente a sistemas con diferentes periodos estructurales de vibración y distintas combinaciones de valores de $\alpha$ y $\gamma$. 
En las figuras 9a y 9b se muestran curvas de excedencia de desplazamiento asociadas a diferentes valores de periodos de vibración para sistemas duales con $\alpha=0.30$ y $\gamma=0.40$ correspondiente a la zona A y $\mathrm{D}$, respectivamente. En las figuras se puede observar que las tasas de excedencia de desplazamiento para la zona $\mathrm{D}$, correspondiente a suelo blando, son mayores que las asociadas a la zona A, correspondiente a suelo firme. Esto hace ver que, para una misma tasa de excedencia anual, a los sistemas ubicados en suelos blandos les corresponde una mayor demanda de desplazamientos.

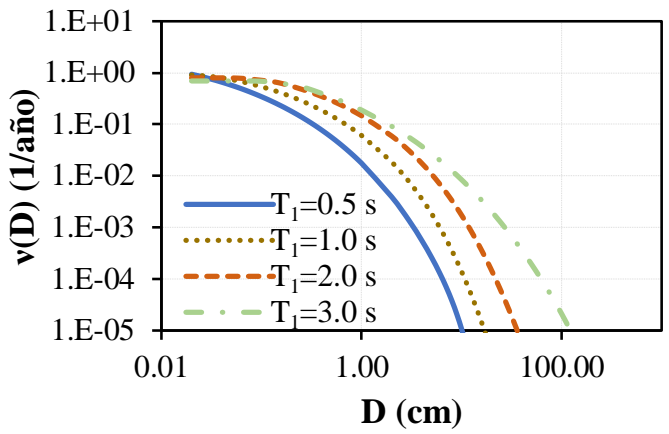

a) Zona $\mathrm{A}$

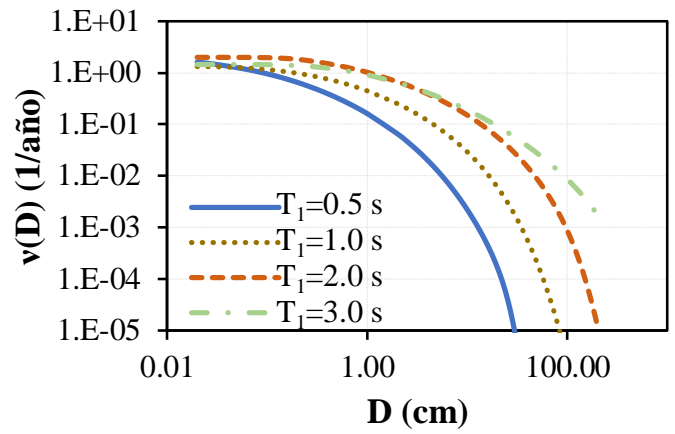

b) Zona D

Figura 9. Curvas de excedencia de desplazamiento para S1GDL dual con $\alpha=0.30$ y $\gamma=0.40$

\section{Influencia de los parámetros $\alpha$ y $\gamma$ en los espectros de Tasa de Excedencia Uniforme (ETEU) de desplazamiento}

Los ETEU de desplazamiento se obtienen a partir de las curvas de excedencia anual de demanda estructural, y contienen las ordenadas máximas que pueden presentarse en un sitio en particular para un periodo de retorno dado, $\mathrm{T}_{\mathrm{r}}$ (el recíproco de la tasa de excedencia, $v$, es el periodo de retorno).

Para este estudio se obtienen los ETEU de desplazamiento asociados a una tasa de excedencia $v=0.004$, la cual equivale a un periodo de recurrencia de 250 años. En las figuras 10 y 11 se muestran, como ejemplo, los ETEU de desplazamiento para sistemas convencionales (línea punteada) y para sistemas duales (líneas continuas), considerando $\alpha=0.30$ y distintos valores de $\gamma$ para las zonas A y D, respectivamente. En las figuras se observa que las demandas de desplazamiento para sistemas ubicados en la zona A (suelo firme) son aproximadamente 10 veces menores que para los sistemas ubicados en la zona D (suelo blando), lo que muestra la importante amplificación de la respuesta que existe en suelos blandos, debido a la presencia de estratos de arcillas muy compresibles. Se puede ver, además, que los ETEU correspondientes a la zona A presentan menor diferencia al hacer variar $\gamma$, comparados con las diferencias correspondientes a los ETEU de la zona D.

Por otro lado, se observa que tanto para la zona A como para la zona D los valores de desplazamiento para los sistemas duales disminuyen conforme aumenta el valor de $\gamma$ para periodos de vibrar menores a 2.6 y $1.8 \mathrm{~s}$, respectivamente; sin embargo, para periodos mayores, este comportamiento se invierte. Este periodo límite, aquí llamado periodo característico $\left(\mathrm{T}_{\mathrm{c}}\right)$, es distinto para cada tipo de suelo. 


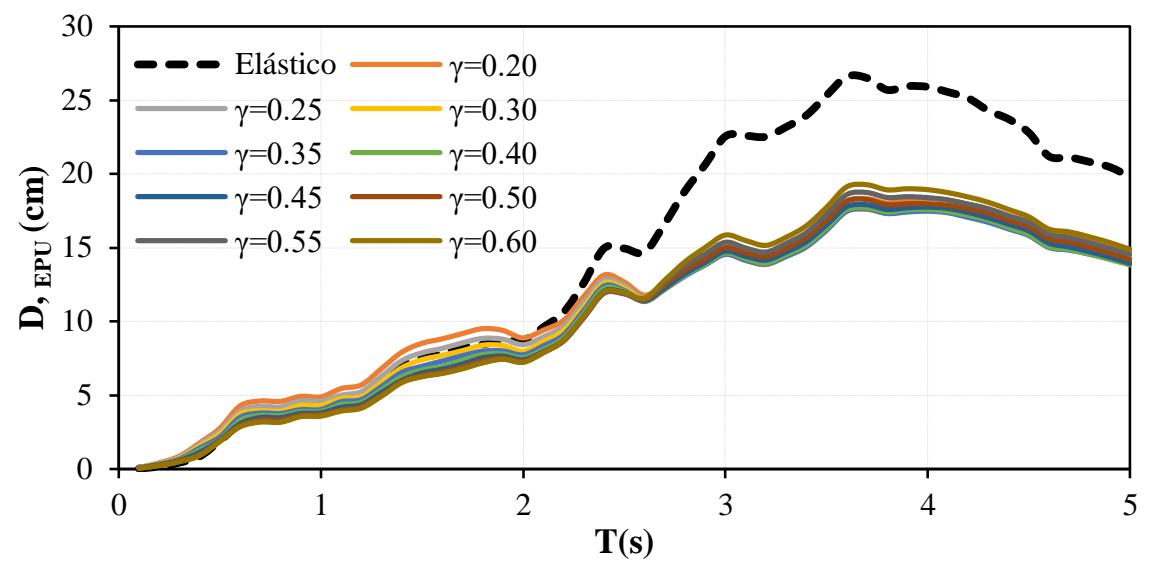

Figura 10. Espectros de tasa de excedencia uniforme para $\alpha=0.30$ y distintos valores de $\gamma$, correspondientes a la zona $\mathrm{A}$

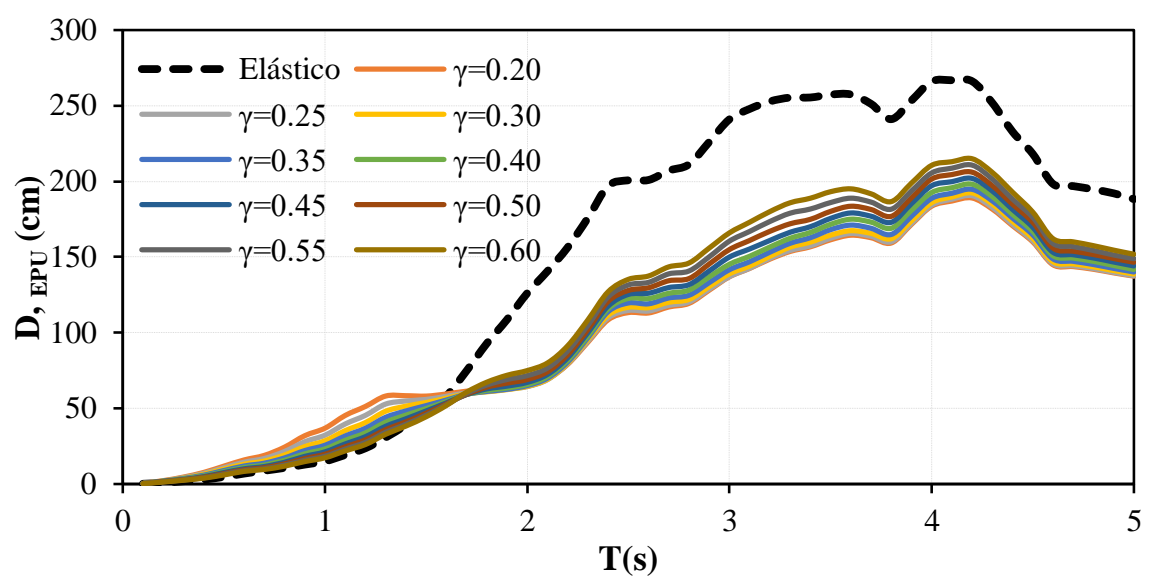

Figura 11. Espectros de tasa de excedencia uniforme para $\alpha=0.30$ y distintos valores de $\gamma$, correspondientes a la zona $\mathrm{D}$

En las figuras 12 y 13 se muestran ETEU de desplazamiento para sistemas convencionales (línea punteada) y sistemas duales (líneas continuas) considerando $\gamma=0.30$ y distintos valores de $\alpha$ para las zonas A y D, respectivamente. En las figuras se observa que los valores de desplazamiento para los sistemas duales disminuyen conforme se aumenta el valor de $\alpha$ para periodos de vibrar menores a $\mathrm{T}_{\mathrm{c}}=2.6$ y $\mathrm{T}_{\mathrm{c}}=1.8 \mathrm{~s}$, respectivamente; sin embargo, para periodos mayores, este comportamiento se invierte.

En las figuras 10 a 13 se observa que la mayor diferencia entre los valores de desplazamientos espectrales se tiene cuando se hace variar el valor $\alpha$, y que en general entre más grande son los valores de $\alpha$ y $\gamma$, el espectro inelástico correspondiente al sistema dual tiende a aproximarse al espectro elástico, debido a que el disipador va dejando de disipar energía (se tienen menores demandas de ductilidad en el disipador), lo que hace que a partir del periodo $T_{c}$ el comportamiento del desplazamiento al aumentar $\alpha$ o $\gamma$ se invierta. 


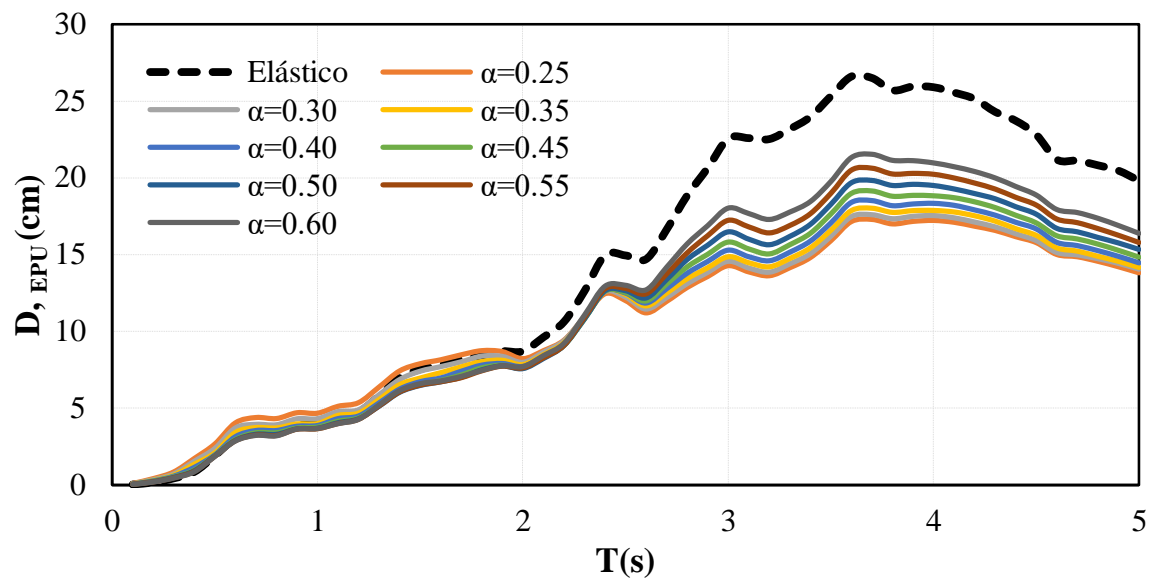

Figura 12. Espectros de tasa de excedencia uniforme para $\gamma=0.30$ y distintos valores de $\alpha$, correspondientes a la zona $\mathrm{A}$

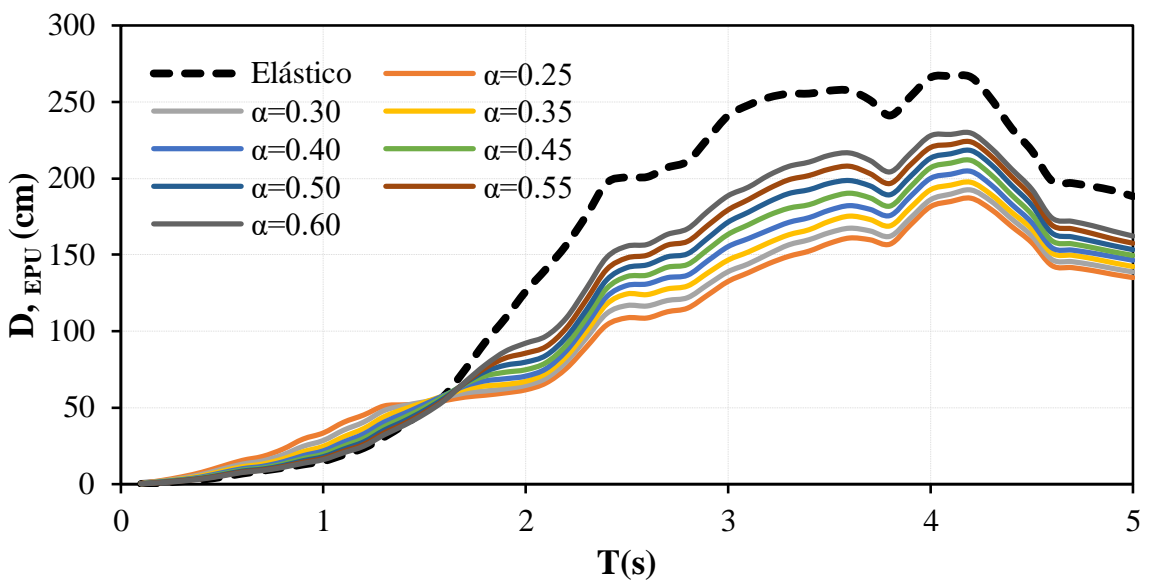

Figura 13. Espectros de tasa de excedencia uniforme para $\gamma=0.30$ y distintos valores de $\alpha$, correspondientes a la zona $\mathrm{D}$

Además, se observa que los ETEU de desplazamiento para los sistemas duales presentan un comportamiento similar a los espectros inelásticos de desplazamiento de sistemas convencionales (sistemas sin disipador) para una demanda de ductilidad constante. Esto se debe a que en ambos casos el comportamiento del sistema estructural es inelástico. La figura 13 muestra que en las zonas sensibles a la aceleración (periodos cortos de vibración), el desplazamiento inelástico es mayor que el desplazamiento elástico, y la diferencia tiende a ser mayor para mayores demandas de ductilidad del sistema (menores valores de $\gamma$ ). Por otro lado, en las zonas sensibles a la velocidad el desplazamiento inelástico puede ser mayor o menor que el desplazamiento elástico debido a que la demanda de ductilidad del sistema afecta al comportamiento estructural de manera irregular; y para la zona sensible al desplazamiento (periodos largos de vibración), los desplazamientos inelásticos tienden a parecerse a los desplazamientos elásticos debido a que la demanda de ductilidad del sistema deja de ser significativa (Chopra, 2012). En resumen, los cocientes de ETEU de desplazamiento inelástico de sistemas duales y ETEU de desplazamiento elástico de sistemas convencionales son mayores que la unidad en el intervalo de periodos de vibración sensibles a la aceleración y parte inicial del intervalo de periodos sensibles a la velocidad, mientras que, los cocientes son menores a la unidad para periodos de vibración mayores, como se muestra más adelante. 
En las figuras 14 y 15 se muestran los ETEU de seudoaceleración para sistemas convencionales (línea punteada) y sistemas duales (líneas continuas) considerando $\gamma=0.30$ y distintos valores de $\alpha$ para las zonas A y $\mathrm{D}$, respectivamente. En las figuras se observa que tanto para la zona A como para la zona D los valores de seudoaceleración correspondientes a los sistemas duales son menores que los elásticos y que disminuyen conforme el valor de $\alpha$ disminuye, en general para periodos mayores a $0.3 \mathrm{~s}$. Los valores de seudoaceleración disminuyen hasta un $85 \%$ con respecto a lo asociado a un comportamiento elástico debido a la disipación de energía que se presenta en el sistema secundario, lo que permite diseñar a los sistemas con amortiguamiento suplementario con fuerzas menores a las elásticas.

\section{Influencia de los parámetros $\alpha$ y $\gamma$ en los cocientes entre ETEU de desplazamiento}

El diseño sísmico de estructuras comúnmente se realiza utilizando espectros de diseño modificados por diferentes factores (i.e., sobre-resistencia, ductilidad, etc.). Cuando la estructura está equipada con disipadores de energía del tipo histerético, el espectro de diseño de desplazamientos se puede modificar, además, mediante un factor (aquí llamado $F_{m d}$ ) que considera la disipación de energía que proporciona el sistema secundario.

Los factores $F_{m d}$ para cada zona se obtienen aquí a partir de cocientes de espectros de tasa de excedencia uniforme (ETEU) de desplazamiento asociados a una tasa media anual de excedencia $v=0.004$, correspondientes a sistemas con disipadores histeréticos y ETEU para sistemas convencionales, suponiendo un porcentaje de amortiguamiento crítico $\xi=5 \%$, es decir:

$F_{m d}=\frac{D_{\text {ETEU }}\left(T_{1}, \alpha, \gamma, \xi=5 \%\right)}{D_{\text {ETEU }}\left(T_{1}, \xi=5 \%\right)}$

donde $D$,ETEU $_{1}\left(T_{1}, \alpha, \gamma, \xi=5 \%\right)$ es el ETEU de sistemas equipados con disipadores de energía de tipo histerético (S1GDL dual), y $D,_{E T E U}\left(T_{1}, \xi=5 \%\right)$ es el ETEU para sistemas convencionales sin disipadores de energía (S1GDL simple).

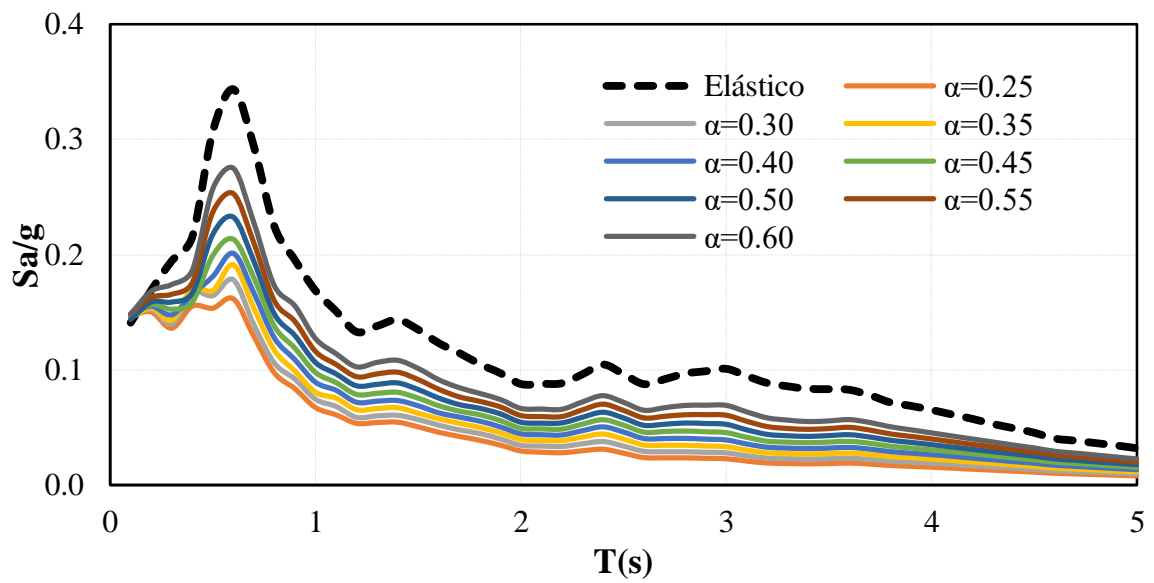

Figura 14. Espectros de seudoaceleración para $\gamma=0.30$ y distintos valores de $\alpha$, correspondientes a la zona $\mathrm{A}$ 


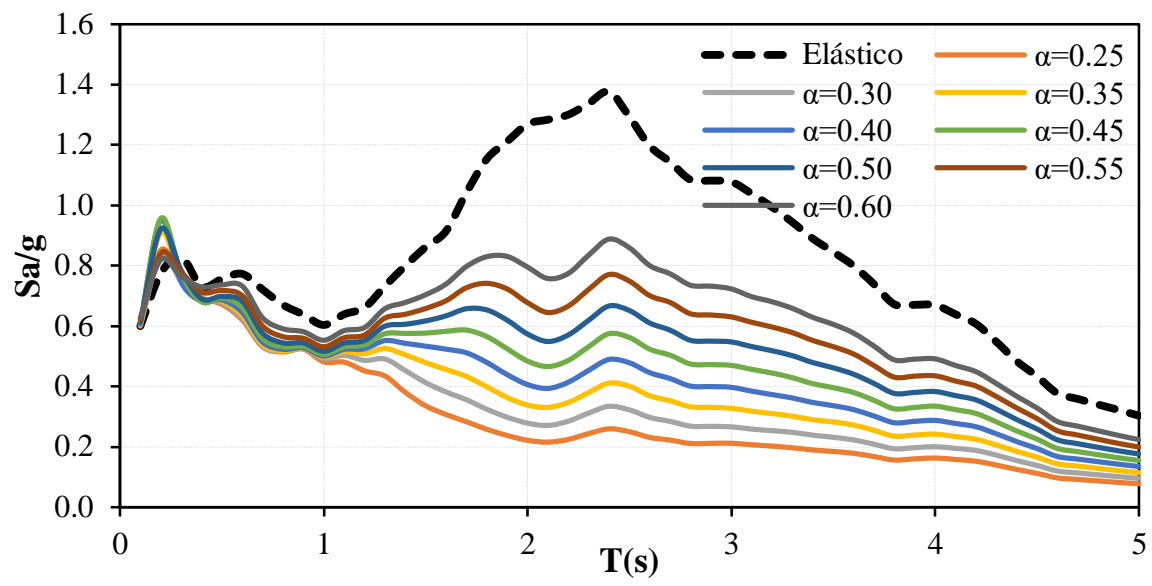

Figura 15. Espectros de seudoaceleración para $\gamma=0.30$ y distintos valores de $\alpha$, correspondientes a la zona $\mathrm{D}$

Los cocientes entre ETEU de desplazamiento se obtuvieron para diferentes combinaciones del sistema dual dado por los parámetros $\alpha$ y $\gamma$ (ver tabla 1) y para las diferentes zonas de la ciudad de México (ver tabla 3). En la figura 16 se muestra la forma de los cocientes de ordenadas espectrales de desplazamiento considerando $\alpha=0.30$ y distintos valores de $\gamma$ para las siete zonas consideradas. En las figuras se observa que el periodo característico $\left(\mathrm{T}_{\mathrm{c}}\right)$ previamente definido, es distinto para cada zona y tienen un valor muy cercano al periodo dominante del suelo para el caso de suelos blandos $\left(\mathrm{T}_{\mathrm{s}}>1.0 \mathrm{~s}\right)$. Para el caso de suelos con $\mathrm{T}_{\mathrm{s}} \leq 1.0$ s correspondientes a las zonas A (suelo firme) y B (suelo intermedio) el periodo característico no tiene relación con el periodo dominante del suelo (porque aquí se define el periodo dominante en función del espectro de aceleración y no del de desplazamiento). En las figuras 16a a 16g se observa que la forma y los valores de los cocientes espectrales son diferentes para cada zona; por lo tanto, las expresiones propuestas en la siguiente sección tienen parámetros diferentes, según el tipo de suelo que se trate.

\section{EXPRESIONES MATEMÁTICAS PROPUESTAS PARA EL FACTOR DE MODIFICACIÓN DE ESPECTROS DE DESPLAZAMIENTOS APLICABLES A LA CIUDAD DE MÉXICO}

Para proponer las expresiones matemáticas, fue necesario primero obtener todos los cocientes entre ETEU de desplazamiento para las diferentes combinaciones del sistema dual dado por los parámetros $\alpha$ y $\gamma$ (ver tabla 1) y para las diferentes zonas consideradas (ver tabla 3). A partir de los cocientes espectrales, se ajustaron mediante el método de mínimos cuadrados, expresiones matemáticas para el factor $F_{m d}$, el cual está en función del periodo dominante de suelo, del periodo de vibrar de la estructura, así como de los parámetros $\alpha$ y $\gamma$. La ecuación 17 muestra las expresiones propuestas para sistemas estructurales equipados con disipadores del tipo histerético, desplantados en distintos tipos de suelo dentro de la Ciudad de México:

$F_{m d}=\left\{\begin{array}{cc}a-b e^{-c\left(\frac{T_{1}}{T_{c}}\right)^{d}}, & T_{s} \leq 0.5 s \\ a+\frac{b\left(\frac{T_{1}}{T_{c}}\right)^{c}}{d+\left(\frac{T_{1}}{T_{c}}\right)^{c}}, & T_{s}>0.5 s\end{array}\right.$

donde $T_{s}$ es el periodo dominante del suelo donde se ubica la estructura; $T_{l}$ es el periodo fundamental de vibrar de la estructura; los valores $a, b, \mathrm{y} c$ están dados por las siguientes ecuaciones: 


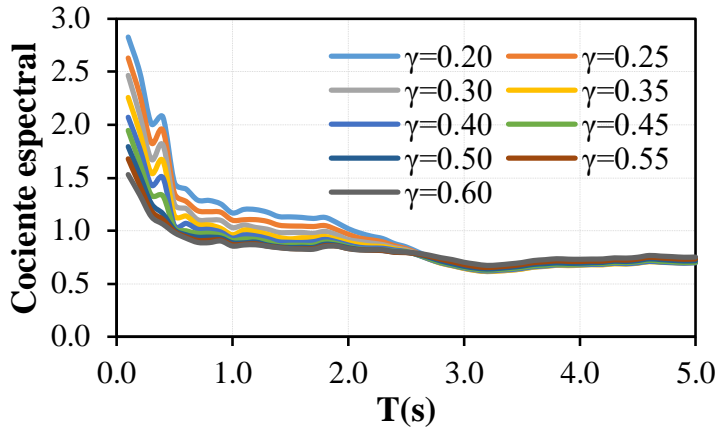

a) Zona $\mathrm{A}$

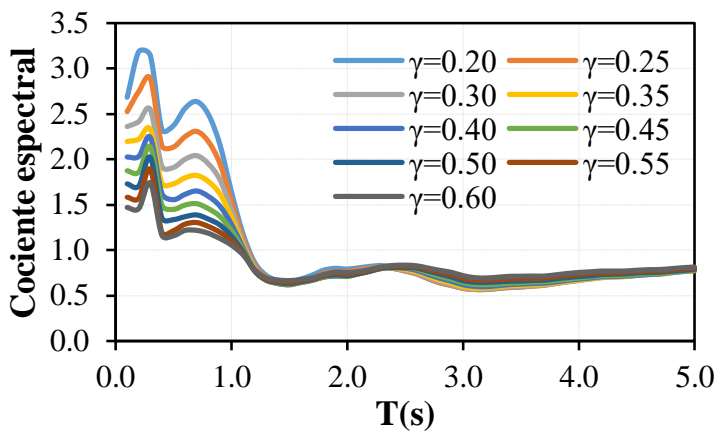

c) Zona C

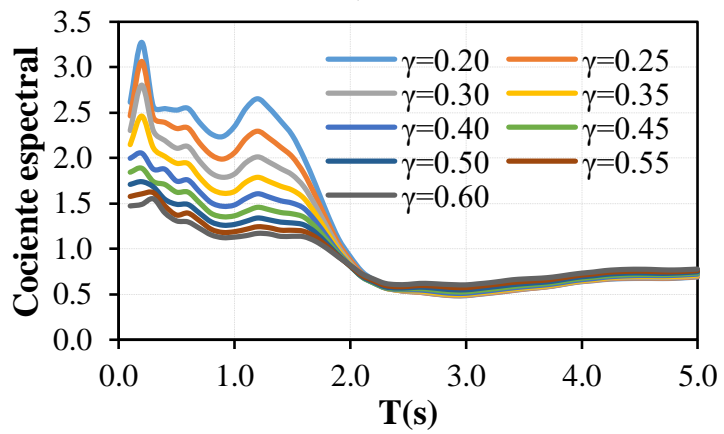

e) Zona E

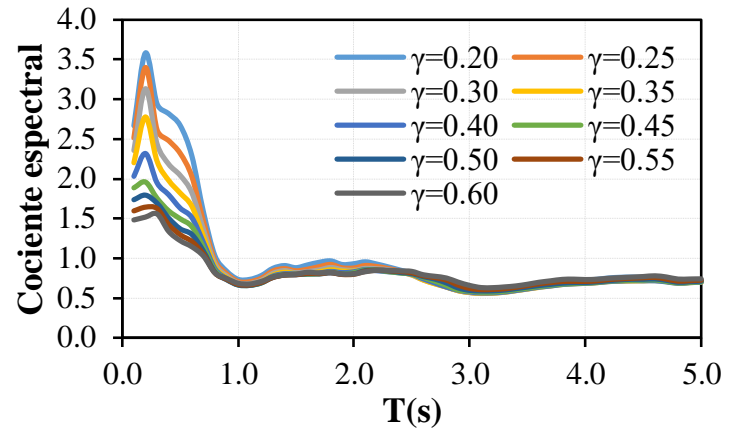

b) Zona B

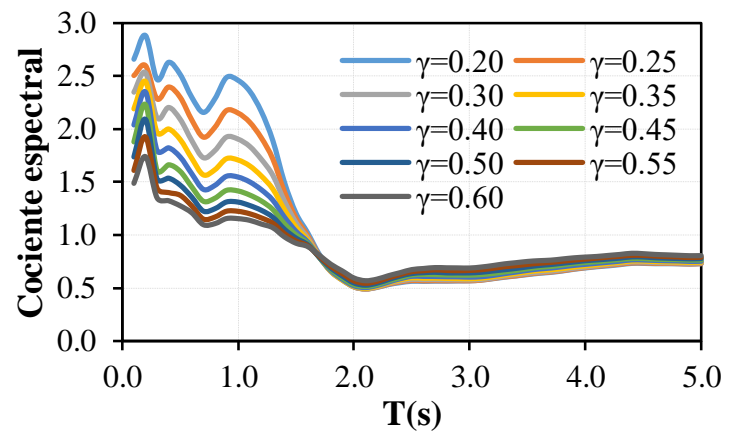

d) Zona D

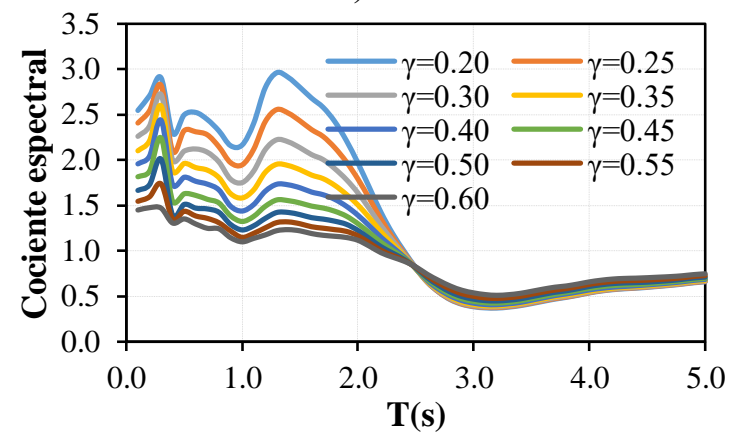

f) Zona F

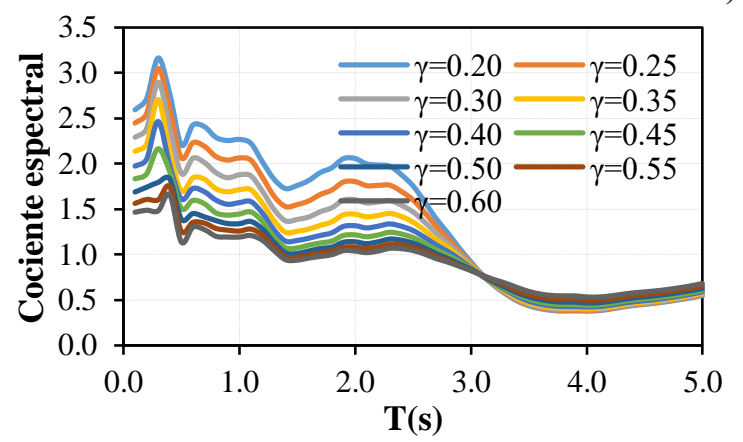

g) Zona G

Figura 16. Cocientes espectrales de desplazamiento para $\alpha=0.30$ y distintos valores de $\gamma$ 


$$
\begin{aligned}
& a=a_{1}+a_{2} \gamma \\
& b=b_{1}+b_{2} \gamma+\frac{b_{3}}{\gamma^{2}} \\
& c=c_{1}+c_{2} \gamma
\end{aligned}
$$

Los valores de los parámetros $a_{1}, a_{2}, b_{1}, b_{2}, b_{3}, c_{1}, \mathrm{y} c_{2}$ de las expresiones propuestas se muestran en la tabla 4. Como ejemplo, en la figura 17 se muestra el ajuste de las expresiones propuestas considerando $\alpha=0.30$ y distintos valores de $\gamma$ para todas las zonas consideradas.

Tabla 4. Valores de los parámetros para estimar el factor $F_{m d}$

\begin{tabular}{cccccccc}
\hline \multirow{2}{*}{ Parámetro } & \multicolumn{7}{c}{ Periodo del sitio $(\mathrm{s})$} \\
\cline { 2 - 8 } & $\mathrm{T}_{\mathrm{s}} \leq 0.5$ & $0.5<\mathrm{T}_{\mathrm{s}} \leq 1.0$ & $1.0<\mathrm{T}_{\mathrm{s}} \leq 1.5$ & $1.5<\mathrm{T}_{\mathrm{s}} \leq 2.0$ & $2.0<\mathrm{T}_{\mathrm{s}} \leq 2.5$ & $2.5<\mathrm{T}_{\mathrm{s}} \leq 3.0$ & $3.0<\mathrm{T}_{\mathrm{s}} \leq 4.0$ \\
\hline $\mathrm{T}_{\mathrm{c}}$ & 2.6 & 2.5 & 1.3 & 1.8 & 2.2 & $2.29+0.71 \alpha$ & $2.82+0.94 \alpha$ \\
$\mathrm{a}_{1}$ & $4.18-0.95 \alpha$ & $0.7+0.08 \alpha$ & $0.56+0.3 \alpha$ & $0.38+0.59 \alpha$ & $0.34+0.59 \alpha$ & $0.25+0.57 \alpha$ & $0.89 \alpha$ \\
$\mathrm{a}_{2}$ & $1.43-13.62 \alpha$ & $-0.22+0.67 \alpha$ & $0.43 \alpha$ & $0.29-0.13 \alpha$ & $0.25+0.05 \alpha$ & $0.12+0.67 \alpha$ & $0.17+0.65 \alpha$ \\
$\mathrm{b}_{1}$ & $3.9-0.82 \alpha$ & $4.4-6.5 \alpha$ & $4.6-7.8 \alpha$ & $4.7-8.5 \alpha$ & $4.31-6.9 \alpha$ & $4.2-6.5 \alpha$ & $6.4-10.6 \alpha$ \\
$\mathrm{b}_{2}$ & $1.85-17.26 \alpha$ & $-4.16+4.94 \alpha$ & $-5.01+7.75 \alpha$ & $-6.71+13.21 \alpha$ & $-5.41+8.42 \alpha$ & $-4.65+6.39 \alpha$ & $-7.57+12.43 \alpha$ \\
$\mathrm{b}_{3}$ & 0 & $0.04-0.07 \alpha$ & $0.03-0.04 \alpha$ & $0.03-0.05 \alpha$ & $0.03-0.04 \alpha$ & $0.06-0.1 \alpha$ & $-0.03+0.06 \alpha$ \\
$\mathrm{c}_{1}$ & $0.16+0.18 \alpha$ & -5.7 & -7.07 & -8.13 & -8.97 & -9.77 & -4.58 \\
$\mathrm{c}_{2}$ & $0.04-0.93 \alpha$ & 0 & 0 & 0 & 0 & 0 & 0 \\
$\mathrm{~d}$ & $-0.92+0.59 \alpha$ & 2161 & 6.35 & 6.8 & 5.32 & 3.49 & 2.22 \\
\hline
\end{tabular}

En la tabla 5 se muestra el valor promedio del coeficiente de determinación $\left(\mathrm{R}^{2}\right)$ de las expresiones matemáticas propuestas, para las diferentes zonas. Este coeficiente refleja la bondad de los ajustes y la variación de los resultados (nivel de error) de las expresiones matemáticas propuestas en este estudio. Por otra parte, las expresiones podrían ser más exactas si se añaden más parámetros con el fin de ajustarse con una mejor aproximación a los datos, pero esto haría que fuesen más complejas; sin embargo, las expresiones aquí propuestas tienen un coeficiente de determinación mayor o igual a 0.70 , por lo que, se considera que tienen suficiente aproximación, y se consideran útiles para el diseño sísmico preliminar de estructuras.

Tabla 5. Coeficiente de determinación

\begin{tabular}{cc}
\hline Zona & $\mathrm{R}^{2}$ \\
\hline $\mathrm{A}$ & 0.82 \\
$\mathrm{~B}$ & 0.84 \\
$\mathrm{C}$ & 0.85 \\
$\mathrm{D}$ & 0.85 \\
$\mathrm{E}$ & 0.88 \\
$\mathrm{~F}$ & 0.88 \\
$\mathrm{G}$ & 0.70 \\
\hline
\end{tabular}




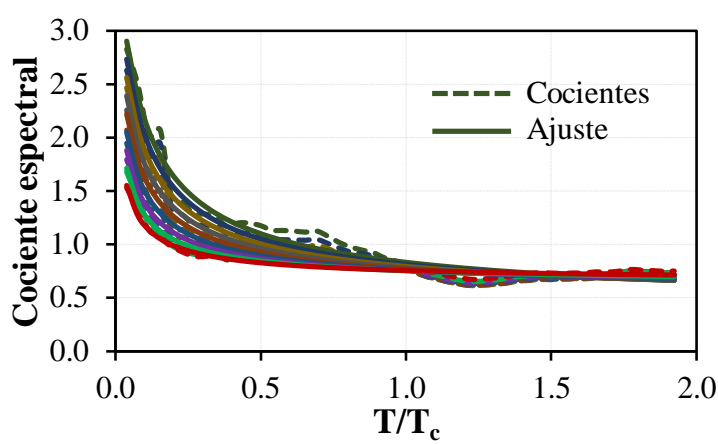

a) Zona $\mathrm{A}$

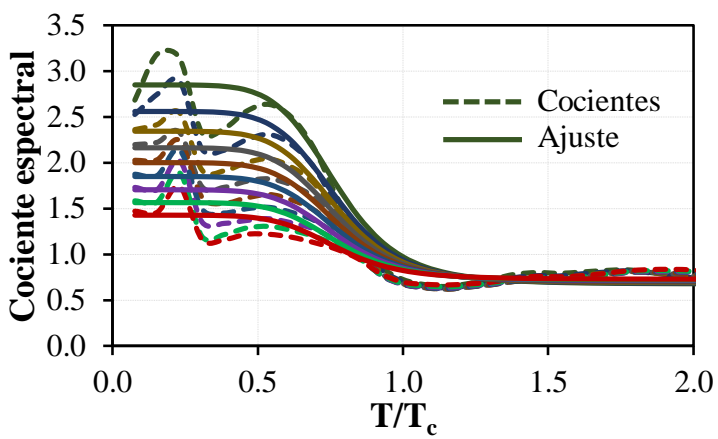

c) Zona $\mathrm{C}$

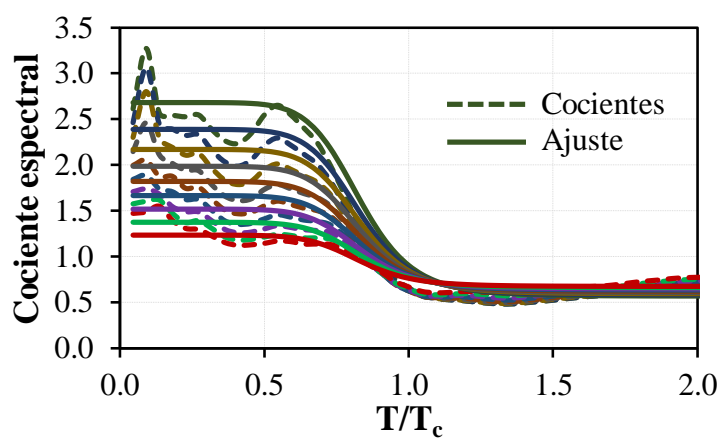

e) Zona E

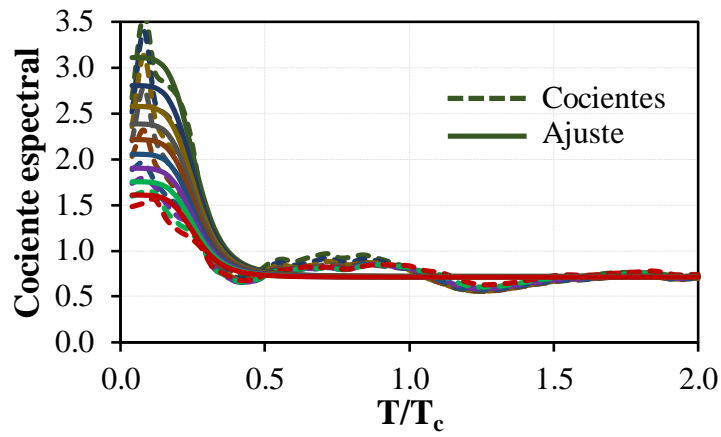

b) Zona B

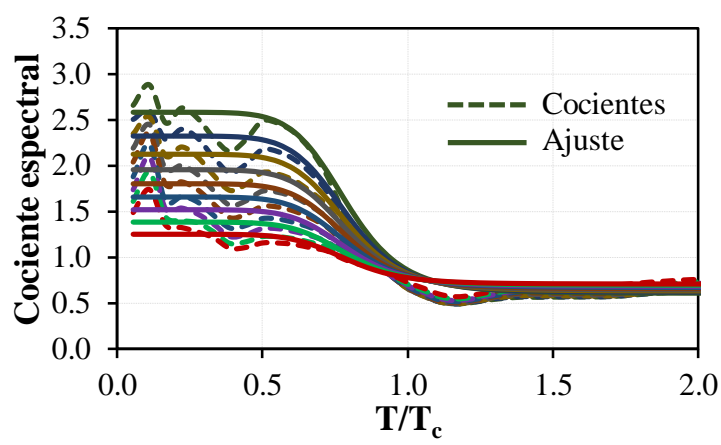

d) Zona D

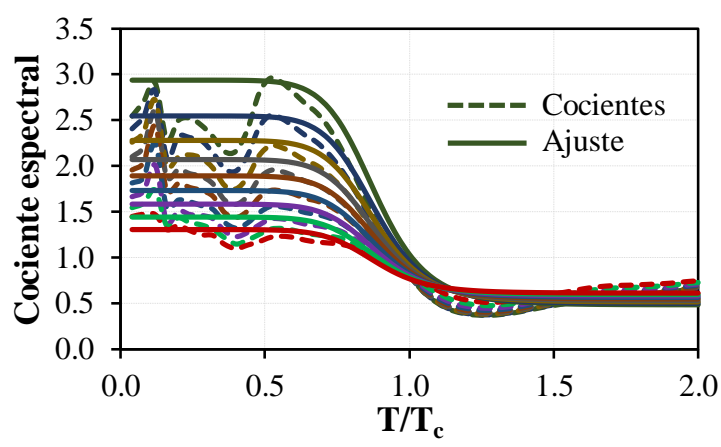

f) Zona $\mathrm{F}$

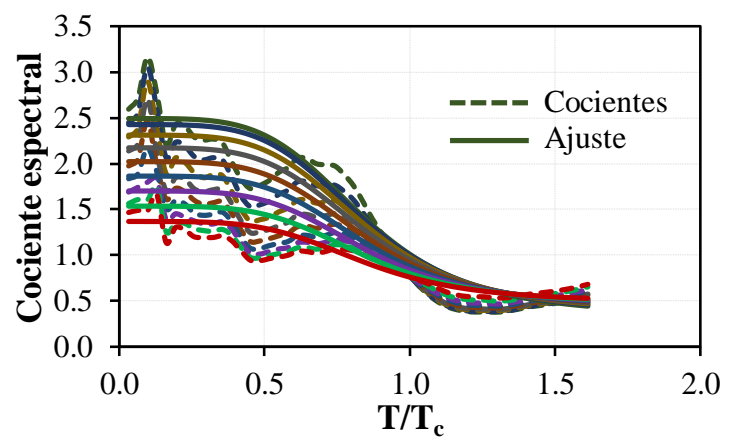

g) Zona G

Figura 17. Comparación entre los cocientes obtenidos con las expresiones propuestas para $\alpha=0.30$ y distintos valores de $\gamma$ 


\section{APLICACIÓN DE LAS EXPRESIONES PROPUESTAS ANTE LA OCURRENCIA DE SISMOS INTRAPLACA}

Las expresiones matemáticas propuestas en las secciones anteriores para el factor de modificación de desplazamiento, aplicable a sistemas estructurales equipados con disipadores del tipo histerético desplantados en distintos tipos de suelo de la ciudad de México, se obtuvieron considerando sismos del tipo subducción; sin embargo, en lo que sigue se verifica que dichas expresiones también son adecuadas para estimar los cocientes de desplazamiento correspondientes a movimientos sísmicos provocados por sismos del tipo intraplaca (como el sismo de Puebla-Morelos que tuvo una magnitud de 7.1, y el sismo ChiautlaPuebla, con magnitud igual a 5.7). El sismo Puebla-Morelos se eligió por provocar las más altas intensidades en la ciudad de México desde el sismo con magnitud igual a 8.1 ocurrido el 19 de septiembre de 1985, lo que ocasionó el colapso de varias estructuras y varias muertes (Galvis et al., 2017; Ruiz et al., 2020), y se registró el día 19 de septiembre de 2017 con epicentro en el límite estatal entre los estados de Puebla y Morelos (Servicio Sismológico Nacional, 2017). Por otro lado, el sismo Chiautla-Puebla (con epicentro al sureste de Chiautla de Tapia, Puebla), a diferencia del sismo de Puebla-Morelos, se eligió por provocar intensidades entre bajas e intermedias, y se registró el día 22 de mayo de 2009. La finalidad de seleccionar estos eventos fue para observar el comportamiento de los cocientes espectrales ante sismos que provocaron movimientos con intensidades tanto altas como intermedias.

En las figuras 18 y 19 se muestran los cocientes de desplazamiento correspondientes al sismo de Puebla-Morelos y al sismo de Chiautla-Puebla, respectivamente; así como, el ajuste de las expresiones propuestas (ec. 17) considerando $\alpha=0.30$ y distintos valores de $\gamma$ para todas las zonas consideradas. Los cocientes de desplazamiento correspondientes al sismo de Puebla-Morelos y al sismo Chiautla-Puebla se obtuvieron utilizando 67 y 64 movimientos sísmicos, respectivamente, registrados en diferentes estaciones localizadas en la ciudad de México, mediante la red acelerográfica del CIRES (Centro de Instrumentación y Registro Sísmico A. C.) y la RAII-UNAM (Instituto de Ingeniería de la Universidad Nacional Autónoma de México).

En las figuras 18 y 19 se puede apreciar que los valores de los cocientes de desplazamiento correspondientes al sismo de Puebla-Morelos, en general, son mayores que los correspondientes al sismo de Chiautla-Puebla para relaciones $\mathrm{T} / \mathrm{T}_{\mathrm{c}}$ menores a 1 ; mientras que para relaciones mayores de $\mathrm{T} / \mathrm{T}_{\mathrm{c}}$, los cocientes de desplazamiento correspondientes a los dos sismos, tienen valores similares. Así mismo, en las figuras 17 y 18 se puede ver que los valores de los cocientes de desplazamiento correspondientes a sismos del tipo subducción, son mayores que los correspondientes al sismo intenso del tipo intraplaca (sismo de Puebla-Morelos) para relaciones $\mathrm{T} / \mathrm{T}_{\mathrm{c}}$ menores a 1 ; mientras que para relaciones mayores de $\mathrm{T} / \mathrm{T}_{\mathrm{c}}$, los cocientes de desplazamiento para los dos tipos de sismos, tienen valores similares. Es decir, las mayores diferencias entre los valores de los cocientes de desplazamiento se presentan en el intervalo de periodos cortos de vibración (zona sensible a la aceleración). 


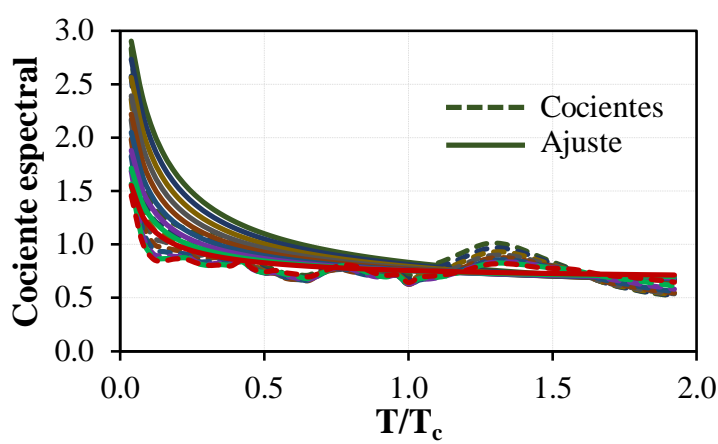

a) Zona $\mathrm{A}$

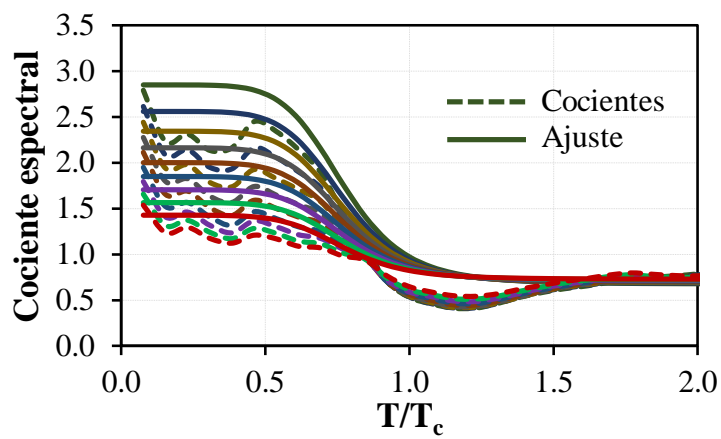

c) Zona $\mathrm{C}$

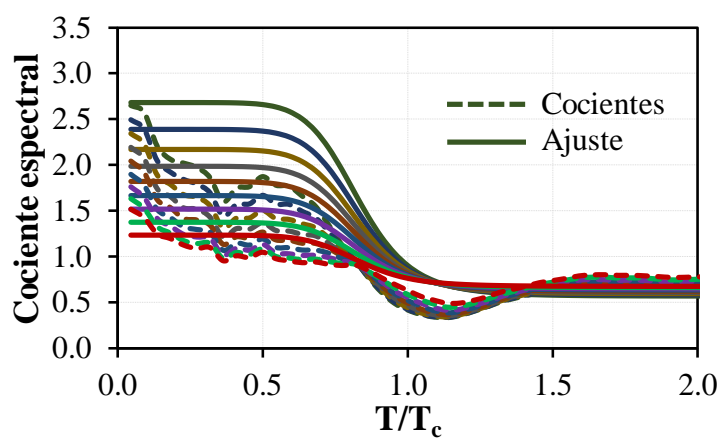

e) Zona $\mathrm{E}$

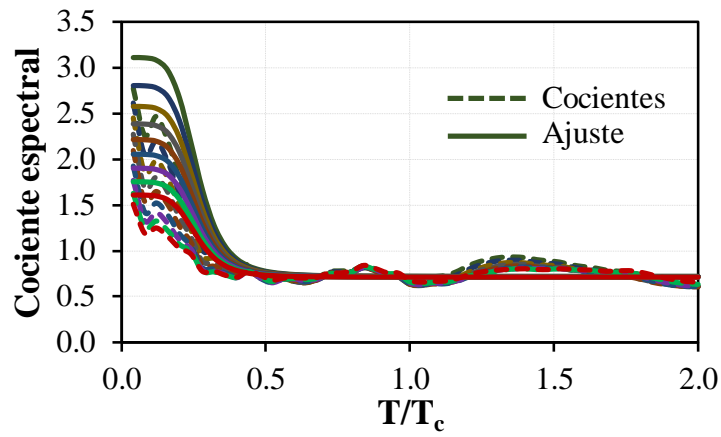

b) Zona B

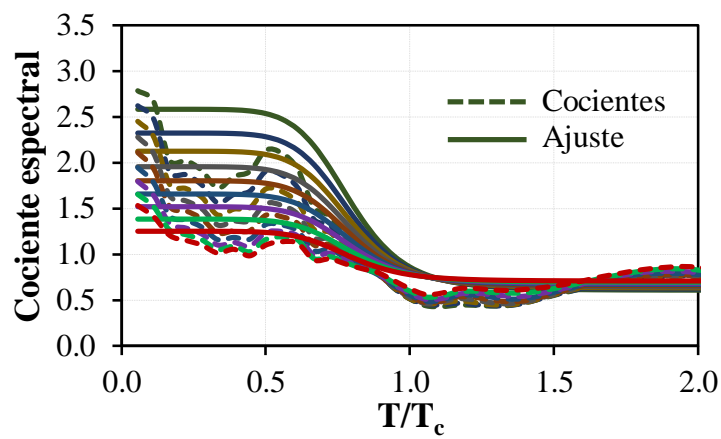

d) Zona D

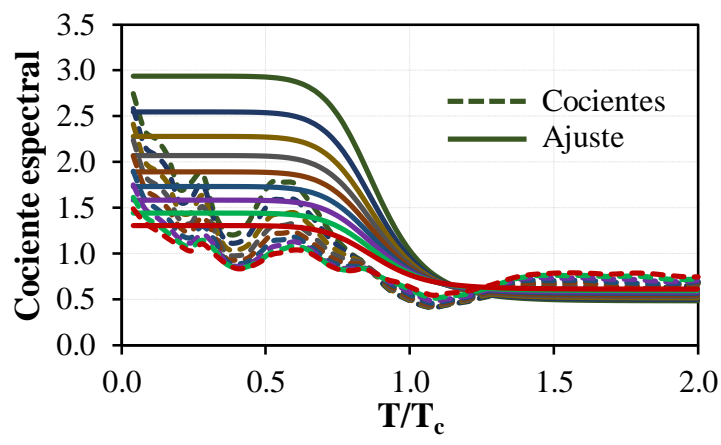

f) Zona $\mathrm{F}$

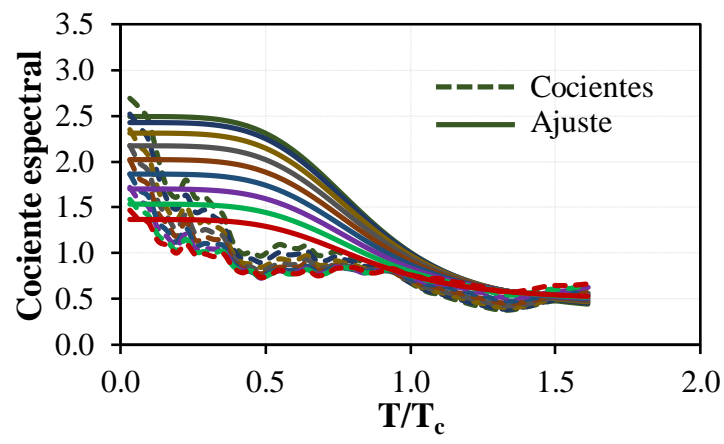

g) Zona G

Figura 18. Comparación entre los cocientes correspondientes al sismo de Puebla-Morelos, y las expresiones propuestas para $\alpha=0.30$ y distintos valores de $\gamma$ 


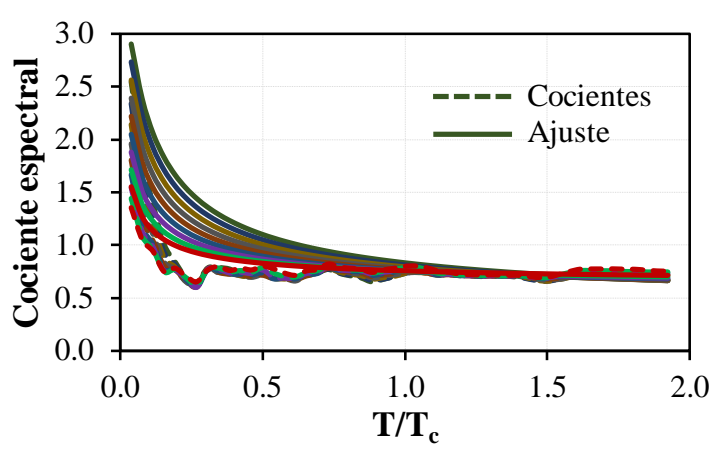

a) Zona $\mathrm{A}$

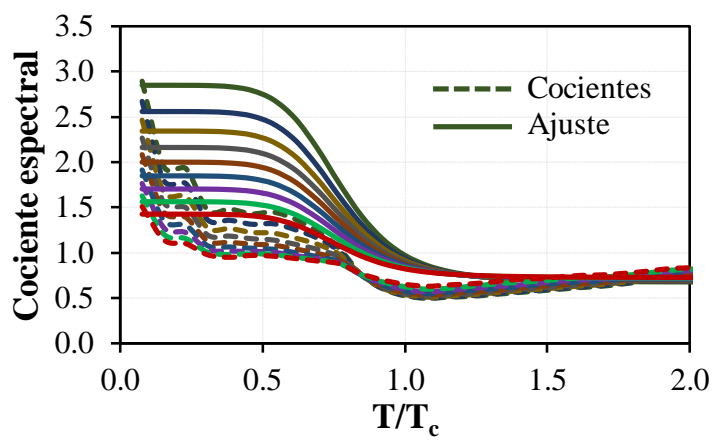

c) Zona $\mathrm{C}$

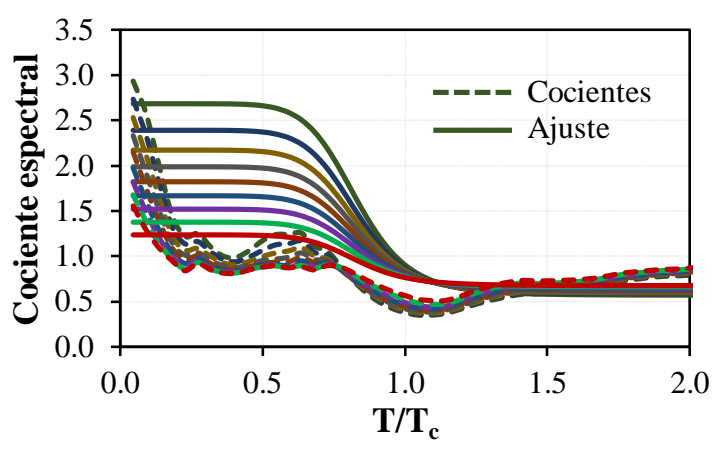

e) Zona E

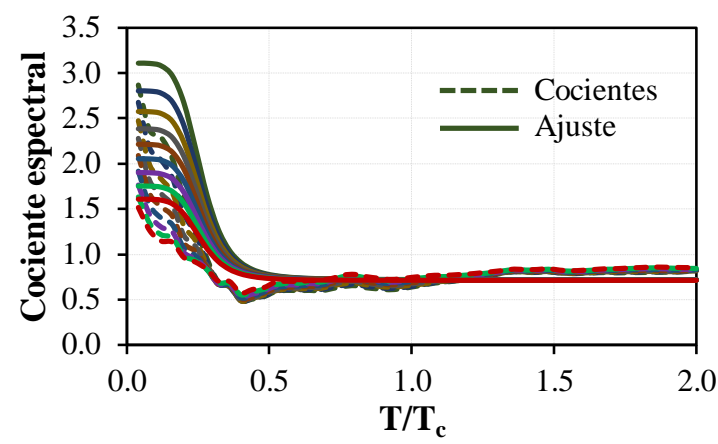

b) Zona B

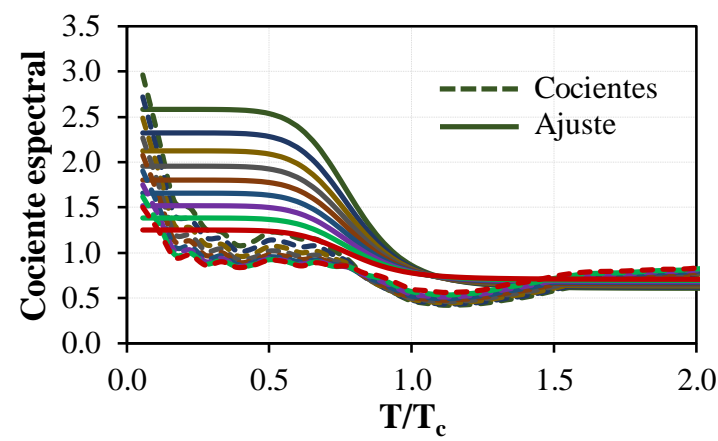

d) Zona D

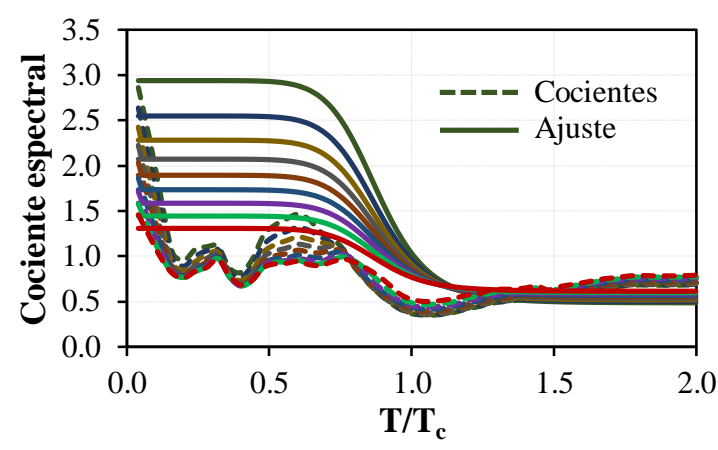

f) Zona $\mathrm{F}$

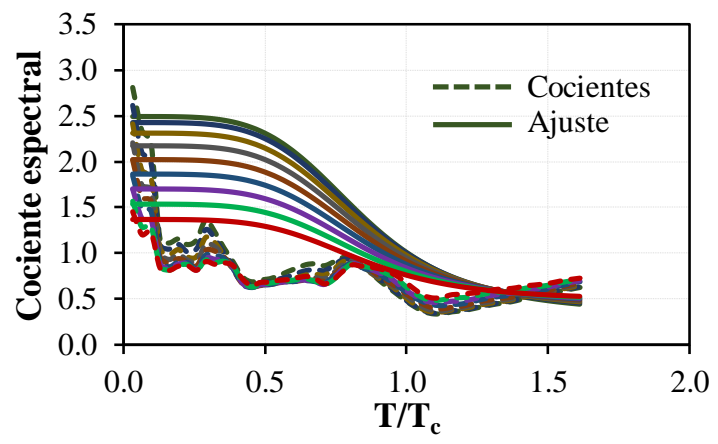

g) Zona $\mathrm{G}$

Figura 19. Comparación entre los cocientes correspondientes al sismo de Chiautla-Puebla, y las expresiones propuestas para $\alpha=0.30$ y distintos valores de $\gamma$ 
De acuerdo con lo anterior, las expresiones matemáticas propuestas para el factor $F_{m d}$, considerando sismos del tipo subducción, resultan más conservadoras para los valores de cocientes de desplazamiento asociados a los sismos del tipo intraplaca aquí analizados, en el intervalo de periodos de vibración sensibles a la aceleración; mientras que para periodos mayores, los factores $F_{m d}$, tienen aproximaciones similares tanto para los valores de cocientes correspondientes a sismos del tipo subducción como para los correspondientes a sismos de tipo intraplaca aquí considerados.

\section{CONCLUSIONES}

\section{Conclusiones generales}

Se aplicó con éxito una metodología basada en un análisis probabilista de riesgo sísmico para obtener expresiones matemáticas de factores $F_{m d}$ que son útiles para modificar las ordenadas espectrales de desplazamiento elástico debido a la presencia en el sistema estructural de disipadores de energía del tipo histérico. La metodología propuesta no se ha aplicado antes a este tipo de sistemas estructurales.

Los factores $F_{m d}$ que se obtuvieron son útiles para estimar la respuesta máxima inelástica de sistemas duales de un grado de libertad y, a partir de esa respuesta, facilitar el diseño estructural preliminar, a través de procedimientos basados en el control de desplazamiento, para sistemas estructurales equipados con disipadores de energía de tipo histerético, localizados en la ciudad de México. Las expresiones resultaron función del periodo de vibrar de la estructura, de las características del sistema dual (estructura-disipador) y del tipo de suelo (duro, intermedio y blando) en donde se ubica el sistema estructural. El estudio considera que el comportamiento no lineal se concentra únicamente en el disipador, el cual no presenta degradación de rigidez ni de resistencia (comportamiento estable) ante cargas alternadas.

Se verificó que los factores $F_{m d}$ pueden aplicarse para el diseño de sistemas estructurales sujetos a movimientos sísmicos causados tanto por eventos sísmicos interplaca, como por eventos intraplaca.

\section{Conclusiones particulares}

- Las expresiones matemáticas propuestas se ajustan adecuadamente para un intervalo de relaciones de rigidez $0.25 \leq \alpha \leq 0.50$ con sus respectivas relaciones de resistencia $\gamma$ propuestas, y para periodos mayores a $0.3 \mathrm{~s}$.

- Se hace ver que, para lograr que el sistema secundario disipe energía es importante seleccionar adecuadamente el intervalo de valores de $\gamma$ en función de $\alpha$ o viceversa, debido a que partir de estos parámetros se puede estimar la demanda de ductilidad en el disipador.

- Entre más grande son los valores de $\alpha$ y $\gamma$, se tienen menores demandas de ductilidad en el disipador; por lo tanto, el espectro modificado tiende a aproximarse al espectro elástico debido a que el disipador disipa menos energía.

- Para cierto valor de $\alpha$ las demandas de desplazamiento disminuyen conforme se aumenta el valor $\gamma$, para periodos estructurales menores al periodo característico $T_{c} ; \sin$ embargo, para periodos mayores a $T_{c}$ este comportamiento se invierte.

- Para cierto valor de $\gamma$ las demandas de desplazamiento disminuyen, conforme se aumenta el valor $\alpha$, para periodos estructurales menores al periodo característico $\mathrm{T}_{c}$; sin embargo, para periodos mayores $\mathrm{a}_{\mathrm{c}}$ este comportamiento se invierte.

- $\quad$ El valor de $T_{c}$ y los valores de los cocientes espectrales de desplazamiento son diferentes para cada tipo de suelo, por lo tanto, la eficiencia del sistema disipador se ve influenciado por las características 
del sitio (periodo dominante) así como por el contenido de frecuencias y la duración efectiva de los diferentes movimientos sísmicos.

\section{AGRADECIMIENTOS}

El presente estudio se realizó dentro de los proyectos PAPIIT IN103517 e IN100320 apoyados por la DGAPA-UNAM. Se agradece al CIRES haber proporcionado los registros sísmicos que se utilizaron en este estudio. El segundo autor agradece al CONACyT el apoyo económico brindado durante sus estudios de posgrado.

\section{REFERENCIAS}

Abdollahzadeh, Banihashemi, Elkaee, y A Esmaeelnia (2012), "Response modification factor of dual moment-resistant frame with buckling restrained brace (BRB)", 15th World Conference on Earthquake Engineering, Lisboa.

Arroyo-Espinoza, D y A Terán-Gilmore (2002), "Factores de reducción de fuerzas sísmicas para el diseño de estructuras con sistemas pasivos de disipación de energía", Revista de Ingeniería Sísmica, Vol. 66, pp. 73-93. DOI: 10.18867/RIS.66.197

Asgarian, B y H R Shokrgozar (2009), "BRBF response modification factor", Journal of Constructional Steel Research, Vol. 65, No.2, pp. 290-298. DOI: 10.1016/j.jcsr.2008.08.002

Avilés, J y L E Pérez-Rocha (1996), "Evaluation of interaction effects on the system period and the system damping due to foundation embedment and layer depth", Soil Dynamics and Earthquake Engineering, Vol. 15, No.1, pp. 11-27. DOI: 10.1016/0267-7261(95)00035-6

Baber, T T y Y K Wen (1981), "Random vibration of hysteretic, degrading systems", Journal of the Engineering Mechanics Division, Vol. 107, No. 6, pp. 1069-1087. DOI: 10.1061/JMCEA3.0002768

Bommer, J J y R Mendis (2004), "Scaling of spectral displacement ordinates with damping ratios", Earthquake Engineering \& Structural Dynamics, Vol. 34, No.2, pp. 145-165. DOI: 10.1002/eqe.414

Bouc, R (1967), "Forced vibration of mechanical system with hysteresis", Proceedings $4^{\text {th }}$ International Conference Nolinear Oscillations, Praga, Checoslovaquia.

Bozzo, L M, J Ramirez, J Bairan, G. Bozzo y E Muñoz (2020), "Precast buildings equipped with SLB seismic devices", 17th World Conference on Earthquake Engineering, Sendai, Japón.

Cameron, J B, N Makris y I D Aiken (2004), "Component testing, seismic evaluation and characterization of buckling-restrained braces", Journal of Earthquake Engineering, Vol. 130, No. 6, pp. 880-894. DOI: 10.1061/(ASCE)0733-9445(2004)130:6(880)

Cameron, W I y R A Green (2007), "Damping correction factors for horizontal ground-motion response spectra", Bulletin of the Seismological Society of America, Vol. 97, No. 3, pp. 934-960. DOI: $\underline{10.1785 / 0120060034}$

Castillo, T y S E Ruiz (2013), "Factor de reducción por amortiguamiento para el diseño sísmico de estructuras con disipadores de energía", Serie Azul 682, Instituto de Ingeniería, UNAM.

Castillo, T y S E Ruiz (2014), "Reduction factors for seismic design spectra for structures with viscous energy dampers", Journal of Earthquake Engineering, Vol. 18, No. 3, pp. 323-349. DOI: $\underline{10.1080 / 13632469.2013 .860932}$ 
Chopra, A K (2012), Dynamics of structures: theory and applications to earthquake engineering, fourth editions, Prentice Hall.

Cornell, C A (1968), "Engineering seismic hazard analysis", Bulletin of the Seismological Society of America, Vol. 58, No. 5, pp. 1583-1606.

Del Valle, E (1988), “Amortiguamiento adicional para reducir efectos sísmicos”, Revista de Ingeniería Sísmica, Vol. 32, pp. 49-76. DOI: 10.18867/RIS.32.323

Esteva, L (1967), “Criterio para la construcción de espectros de espectros para diseño sísmico”, III Simposio panamericano de Estructuras, Caracas, Venezuela.

Foti, D, L Bozzo y F López-Almansa (1998), "Numerical efficiency assessment of energy dissipators for seismic protection of buildings", Earthquake Engineering \& Structural Dynamics, Vol. 27, No. 6, pp. 543-556. DOI: 10.1002/(SICI)1096-9845(199806)27:6<543::AID-EQE733>3.0.CO;2-9

Gaceta Oficial de la Ciudad de México (2017), Reglamento de Construcciones para el Distrito Federal, Ciudad de México.

Galvis, F, E Miranda, P Heresi, H Dávalos y J Ramón Silos (2017), "Preliminary statistics of collapsed buildings in Mexico City in the September 19, 2017 Puebla-Morelos Earthquake", Stanford University.

Guerrero, H, T Ji, A Terán-Gilmore y J A Escobar (2016), “A method for preliminary seismic design and assessment of low-rise structures protected with buckling restrained braces", Engineering Structures, Vol. 123, pp. 141-154. DOI: 10.1016/j.engstruct.2016.05.015

Hidalgo, J P y S E Ruiz (2010), "Factor de reducción $\beta$ por incremento de amortiguamiento viscoso, para estructuras desplantadas sobre roca", Revista de la Sociedad Mexicana de Ingeniería Sísmica, Vol. 83, pp. 1-23. DOI: $10.18867 /$ RIS.83.142

Hurtado, F y L M Bozzo (2008), "Numerical and experimental analysis of a shear-link energy dissipator for seismic protection of buildings", 14th World Conference on Earthquake Engineering, Beijing, China.

Jara, J M, A G Ayala y E Miranda (2000), "Seismic behaviour of structures with energy dissipating systems in Mexico", 12th World Conference on Earthquake Engineering, Auckland, Nueva Zelanda.

Naeim, F y C A Kircher (2001), "On the damping adjustment factors for earthquake response spectra", The Structural Design of Tall Buildings, Vol. 10, No. 5, pp. 361-369. DOI: 10.1002/tal.180

Nakashima, M, K Saburi y B Tsuji (1996), "Energy input and dissipation behavior of structures with hysteretic dampers", Earthquake Engineering \& Structural Dynamics, Vol. 25, No. 5, pp. 483-496. DOI: 10.1002/(SICI)1096-9845(199605)25:5<483::AID-EQE564>3.0.CO;2-K

Nangullasmú, H (2011), "Propuesta de criterios de diseño sísmico conforme al reglamento para marcos no dúctiles de concreto reforzado con disipadores histeréticos", Tesis de Maestría, División de Ciencias Básicas e Ingeniería, UAM, México.

Nuzzo, I, D Losanno y N Caterino (2019), "Seismic design and retrofit of frame structures with hysteretic dampers: a simplified displacement-based procedure", Bulletin of Earthquake Engineering, Vol. 17, pp. 2787-2819. DOI: $10.1007 / \mathrm{s} 10518-019-00558-8$

Ordaz, M y C Montoya (2014), “Programa DEGTRA, Versión 9.3.2”, Programa de cómputo, Instituto de Ingeniería, UNAM, México.

Orellana, M A, S E Ruiz y A Rodríguez-Castellanos (2021), "Displacement Spectra Damping Factors for Preliminary Design of Structures with Hysteretic Energy-Dissipation Devices", Journal of Earthquake Engineering. DOI: 10.1080/13632469.2021.1913455 
Priestley, M J N, G M Calvi y M J Kowalsky (2007), Displacement-based design of structures, Pavia, Italia: IUSS PRESS.

Reglamento de Construcciones para el Distrito Federal (2020), Normas Técnicas Complementarias para Diseño por Sismo, Gaceta Oficial de la Ciudad de México, México.

Rosenblueth, E y L Esteva (1972), "Reliability basis for some Mexican codes”, ACI, SP-31, pp. 1-41.

Ruiz, S E y H Badillo (2001), "Performance-based design approach for seismic rehabilitation of buildings with displacement-dependent dissipators", Earthquake Spectra, Vol. 17, No. 3, pp. 531-548. DOI: $\underline{10.1193 / 1.1586187}$

Ruiz Gómez, S E, R Jiménez Jordán, M A Santos Santiago y M A Orellana Ojeda (2020), "Evaluación de la fragilidad de dos soluciones de rehabilitación para un edificio con planta baja débil dañado durante el sismo 19/S17”, Revista de Ingeniería Sísmica, No. 102, pp. 1 -25. DOI: 10.18867/RIS.102.513

Segovia, V y S E Ruiz (2017), "Direct displacement-based design for buildings with hysteretic dampers, using best combinations of stiffness and strength ratios", Journal of Earthquake Engineering, Vol. 21, No. 5, pp. 752-775. DOI: 10.1080/13632469.2016.1185054

Servicio Sismológico Nacional (2017), "Reporte Especial: Sismo del día 19 de Septiembre de 2017, PueblaMorelos (M 7.1)", Universidad Nacional Autónoma de México (UNAM), Ciudad de México, México.

Shome, N y C A Cornell (1999), "Probabilistic Seismic Demand Analysis of Nonlinear Structures", Report No. RMS-35, Department of Civil and Environmental Engineering, Stanford University, Stanford, USA.

Silva-González, F L (1998), "Calibración del método de linealización equivalente estocástica para sistemas histeréticos simples", Tesis de Maestría, División de Estudios de Posgrado, UNAM, México.

Sues, R H, S T Mau y Y K Wen (1988), "Systems identification of degrading hysteretic restoring forces", Journal of Engineering Mechanics, Vol. 114, No. 5, pp. 833-846. DOI: 10.1061/(ASCE)07339399(1988)114:5(833)

Terán-Gilmore, A y N Virto-Cambray (2009), "Preliminary design of low-rise buildings stiffened with buckling-restrained braces by a displacement-based approach", Earthquake Spectra, Vol. 25, No. 1, pp. 185 -211. DOI: 10.1193/1.3054638

Vargas, R y M Bruneau (2009), "Analytical response and design of buildings with metallic structural fuses I", Journal of Structural Engineering, Vol. 135. No. 4, pp. 386-393. DOI: 10.1061/(ASCE)07339445(2009)135:4(386) 